\title{
FETAL SULCATION AND GYRIFICATION IN COMMON MARMOSETS (CALLITHRIX JACCHUS) OBTAINED BY EX VIVO MAGNETIC RESONANCE
}

IMAGING

\section{K. SAWADA, ${ }^{1, *, A}$ K. HIKISHIMA, ${ }^{2,3, A}$ A. Y. MURAYAMA, ${ }^{2,5}$ H. J. OKANO, ${ }^{4}$ E. SASAKI $^{3}$ AND H. OKANO ${ }^{2,5,6, *}$}

${ }^{1}$ Department of Physical Therapy, Faculty of Medical and Health Sciences, Tsukuba International University, Tsuchiura, Japan

${ }^{2}$ Department of Physiology, Keio University School of Medicine, Tokyo, Japan

${ }^{3}$ Central Institute for Experimental Animals, Kawasaki, Japan

${ }^{4}$ Division of Regenerative Medicine, Jikei University School of Medicine, Tokyo, Japan

${ }^{5}$ RIKEN Keio University Joint Research Laboratory, RIKEN Brain Science Institute, Wako, Japan

${ }^{6}$ Keio University Joint Research Laboratory, Brain Science Institute RIKEN, Wako, Saitama Japan

*Correspondence to:

Hideyuki Okano, Department of Physiology, Keio University School of Medicine, 35

Shinanomachi, Shinjuku, Tokyo 160-8582, Japan.

Tel: +81-3-5363-3746, Fax: +81-3-3357-5445 E-mail: hidokano@a2.keio.jp

Kazuhiko Sawada, Department of Physical Therapy, Faculty of Medical and Health Sciences, 
Tsukuba International University, Tsuchiura, Ibaraki 300-0051, Japan

Tel: +81-29-883-6032,E-mail: k-sawada@tius.ac.jp

${ }^{\mathrm{a}}$ These authors contributed equally to this work. 
Abstract - The present study characterized fetal sulcation patterns and gyrification in the cerebrum of the New World monkey group, common marmosets, using a 3D $\mathrm{T}_{2}$-weighted high-resolution anatomical MRI sequence from the fixed brain at 7-tesla ex vivo. Fetal sulcation in the marmoset cerebrum began to indent the lateral fissure and hippocampal sulcus on gestational week $(\mathrm{GW}) 12$, and then the following sulci emerged: the callosal and calcarine sulci on GW 15; the superior temporal sulcus on GW 17; and the circular and occipitotemporal sulci on GW 18. The degree of cortical convolution was evaluated quantitatively based on 2D MRI slices by the gyrification index (GI) and based on 3D MRI data by sulcation index (SI). Both the mean GI and SI increased from GW 16, and were closely correlated with the cortical volume and the cortical surface area during fetal periods (their correlation coefficients marked more than 0.95). After birth, both the mean GI and SI decreased slightly by 2 years of age, whereas the cortical volume and surface area continuously increased. Notably, histological analysis showed that the outer subventricular zone in non-sulcal regions was thicker than that in the presumptive calcarine sulcal region on GW 13, preceding the infolding of the calcarine sulcus. The present results showed definite sulcal infolding on the cerebral cortical surface of the marmosets, with similar pattern and sequence of their emergences to higher-order other primates such as macaques and humans. Differential expansion of the oSVZ may be involved in gyral convolution and sulcal infolding in the developing cerebrum.

Key words: basal radial glia, common marmoset, gyrification, non-human primates, outer subventricular zone

Abbreviations: $\quad$ cal $=$ calcarine sulcus; cas $=$ callosal sulcus; $c c=$ corpus callosum; $\operatorname{cgs}=$ cingulate sulcus $;$ cirs $=$ circular sulcus $;$ cs $=$ central sulcus; $\mathrm{CP}=$ cortical plate; his $=$ 
hippocampal fissure; iSVZ = inner subventricular zone; IZ = intermediate zone; lf = lateral fissure; $\mathrm{MZ}=$ marginal zone; $\mathrm{oSVZ}=$ outer subventricular zone; ots = occipitotemporal sulcus; pos $=$ parietooccipital sulcus $; \mathrm{Pe}=$ a petrous portion of the temporal bone; $\mathrm{PH} 3=$ phosphohistone $\mathrm{H} 3 ; \mathrm{sc}=$ superior colliculus; $\mathrm{SP}=$ subplate; $\mathrm{VZ}=$ ventricular zone 


\section{INTRODUCTION}

The common marmoset (Callithrix jacchus) is a small laboratory primate belonging to the New World monkey group, and exhibits a high sociality with abundant vocal communication, making the use of this animal appropriate for studies of higher cognitive function (Epple 1968) and modeling human behaviors (Hashimoto et al., 2009, Yamazaki et al., 2011a, Yamazaki et al., 2011b). In fact, several studies have been reported with regard to models of cognitive and neurological disorders in marmosets (Iwanami et al., 2005a, b; Fujiyoshi et al., 2007; Yamada et al., 2008; Kobayashi et al., 2012).

A great majority of primate species have a cerebral cortex with a convoluted surface, sulci and gyri. The development of the cerebral sulci has been reported in humans and nonhuman primates by anatomic (Chi et al., 1977; Naidich et al., 1994; Fukunishi et al., 2006; Kashima et al., 2008), sonographic (Naidich et al., 1994), and magnetic resonance imaging (MRI) (Naidich et al., 1994; Prayer et al., 2006; Sawada et al., 2009) studies and three-dimensional (3D) reconstruction of the cortical surface based on MRI (Dubois et al. 2008). Such a fetal sulcation pattern is phylogenetically conserved among primate species (Sawada et al., 2012a). The New World monkey group including the marmosets also has a gyrencephalic cerebrum, while the degree of their cortical convolution is less developed than that in higher order primates, i.e., the Old World monkey group, apes and human (Zilles et al., 1988, 1989). However, the developmental pattern of cerebral sulci in the New World monkey group has not yet been addressed. The aim of this study was to characterize the fetal sulcation pattern of common marmosets. We used the $3 \mathrm{D} \mathrm{T}_{2}$-weighted high-resolution anatomical MRI sequence from the fixed brain of the common marmoset fetuses at 7-tesla, which had been acquired in our recent report (Hikishima et al., 2013).

To correlate the anatomical MRI sequence on fetal sulcation and gyrification with 
histological findings, we paid close attention to the structure, called the outer subventricular zone (oSVZ). The oSVZ was first identified in the human fetal brain as the second proliferative zone in the cerebral cortex and contains many self-renewable progenitor cells called basal radial glia (bRG) (Hansen et al., 2010; Fietz et al., 2010), which is likely to serve to thicken cerebral cortex in gyrencephalic mammals (Lui et al., 2011). We recently found that oSVZ appeared between E85 and E92 (Kelava et al., 2012) in the marmoset, the gestation period of which is 21 weeks. By histological analysis, we showed that the oSVZ in the non-sulcal (convoluted) region was found to be thicker than that in the presumptive calcarine sulcal region on GW 13 of marmoset. Though the common marmosets are generally considered to have a near-lissencephalic cerebrum, we obtained intriguing findings related to the gyrification mechanism by investigating the morphological changes in the neocortex in the early stages of sulcal development in this primate.

\section{EXPERIMENTAL PROCEDURES}

\section{MRI procedures}

The present study used the 4\% paraformaldehyde-fixed brains of common marmosets at each gestational week (GWs) $11(\mathrm{n}=1), 12(\mathrm{n}=1), 13(\mathrm{n}=1), 14(\mathrm{n}=1), 15(\mathrm{n}=1), 16(\mathrm{n}=1), 17(\mathrm{n}=1)$, $18(\mathrm{n}=1), 19(\mathrm{n}=1)$, and postnatal day $(\mathrm{PD}) 0(\mathrm{n}=2)$, and adult marmosets $(2$ years of age $)(\mathrm{n}=$ 1) for evaluating sulcation and gyrification. These were the same samples that had been used previously (Hikishima et al., 2013). Marmoset fetuses were obtained from six breeding pairs of animals purchased from CLEA Japan (Tokyo, Japan) by Cesarean section. For Cesarean section, the mothers were anesthetized by intramuscular administration of $70 \mathrm{mg} / \mathrm{kg} \mathrm{ketamine}$ 
(Sankyo Lifetech Co. Ltd., Tokyo, Japan) and $0.1 \mathrm{mg} / \mathrm{kg}$ atropine sulfate (Mitsubishi Tanabe Pharma Corporation, Osaka, Japan). The animals were then anesthetized with 1.0-3.0\% isoflurane (Abbott Japan, Tokyo, Japan) via a ventilation mask. During the operation, anesthesia was maintained by spontaneous respiration, and both heart rate and arterial oxygen saturation were monitored. Twenty-four embryonic and fetal marmosets ranging in age from GW8-19 (two embryos per each GW) were obtained and immediately euthanized in a humane manner via deep anesthesia. Adult and neonatal (PD 0) animals were euthanized via deep anesthesia (intravenous sodium pentobarbital, $100 \mathrm{mg} / \mathrm{kg}$ ) for highresolution ex vivo MRI. All specimens were then immersed in 4\% paraformaldehyde/ phosphate-buffered saline for 2 weeks. After fixation specimens were stored for 2 weeks in phosphate-buffered saline containing $0.5 \%$ sodium azide and the contrast agent of gadopentetate dimeglumine, $1 \mathrm{mM}$ Magnevist (Schering, Berlin, Germany) (Newman et al., 2009). Ex vivo MRI scans were performed using a 7 tesla Biospec 70/16 MRI scanner (Bruker Biospin GmbH; Ettlingen, Germany) equipped with actively shielded gradients at a maximum strength of $700 \mathrm{mT} / \mathrm{m}$. 3D $\mathrm{T}_{2}$-weighted MRI was acquired by relaxation enhancement (RARE) using the following parameters: effective echo time (TE), $18 \mathrm{~ms}$; repetition time (TR), $250 \mathrm{~ms}$; number of averages (NA), 6; RARE factor, 4 (Hikishima et al., 2013). Specimen and data acquisition specifications are detailed in Table 1.

$<<<$ Table 1 near here $>>>$

\section{D volume-rendered images}

All MR images were incorporated into the 3D reconstruction. The cerebrum was semi-automatically segmented on MR slices using "Morpho" tool of SliceOmatic software 
ver 4.3 (TomoVision, Montreal, Canada) based on image contrast as well as user knowledge of anatomy. The segmented images were then analyzed using the 3D-rendering module of the same software, and cerebral images were rendered in 3D using a surface projection algorithm, which best visualized the surface and sulci of the cerebrum. The 3D-rendered images were then rotated and manipulated in a manner that best visualized the brain morphology by a linear registration method using SliceOmatic software.

\section{Identification of sulci}

The criteria for identifying a sulcus were referenced with the Stereotaxic Atlas of the marmoset Brain (Yuasa et al., 2010). A clear indentation at the cerebral surface with curvature of the adjacent subcortical white matter was considered the earliest indication of a sulcus. Using such criteria, small indentations of the cerebral sulci could be defined. It is impossible to identify such sulci from the pial surface alone, however, since blood vessels can also create indentations in this surface. Thus, digital surface analysis techniques that attempt to identify secondary sulci based on measured surface curvature will fail (Fig. 1).

$<<<$ Figure 1 near here $>>>$

\section{Volume and surface area of cerebral cortex}

All MR images were used for volumetric analysis. The cortical plate (CP) and subplate (SP) were distinguishable from the underlying white matter and/or intermediate zone based on image contrast in our MRI images (Hikishima et al., 2013). These two layers were 
semi-automatically segmented on the MRI images using the "Segmentation editor" tool of Amira ver 5.2 (Visage Imaging, Inc., San Diego, CA, USA) based on image contrast as well as user knowledge of anatomy. According to a procedure of our previous study (Sawada et al. 2010), the cortical volume was calculated by multiplying the summed areas of the CP plus SP by the slice thickness $(100 \mu \mathrm{m})$ using the same software. The surface area of the cortex including sulcal grooves was the computed from 3D MRI images using SliceOmatic software 4.3 .

\section{Gyrification index and sulcation index}

The degree of cortical convolution was evaluated quantitatively based on 2D MRI slices by the gyrification index (GI) according to the procedure by Zilles et al. $(1988,1989)$, and based on 3D MRI data by sulcation index (SI) calculated by a modified procedure originally proposed by Dubois et al. (2008). Procedures and formulas for calculation of the GI and SI were shown in Figure 7 (A, B).

\section{Histology}

A fresh specimen of fetal marmoset brain on GW13 $(n=2)$ was fixed with $4 \%$ PFA at $4^{\circ} \mathrm{C}$ for 24 hours, immersed in $30 \%$ sucrose in PBS at $4^{\circ} \mathrm{C}$ overnight, and embedded in Tissue-Tek and frozen. The frozen block was sectioned serially in the coronal plane at 12-16 $\mu \mathrm{m}$ using a cryostat. Sections were stained with hematoxylin and eosin (HE). Cryosections were washed in $0.3 \%$ Triton-X 100 for $10 \mathrm{~min}$ at room temperature and antigen retrieval was performed in $10 \%$ glycerol / $0.01 \mathrm{M}$ sodium citrate buffer $(\mathrm{pH} 6.0)$ for 1 hour at $70{ }^{\circ} \mathrm{C}$ in a water bath, and then left standing for $30 \mathrm{~min}$ at room temperature. The sections were permeabilized in $0.3 \%$ 
Triton-X 100 for $30 \mathrm{~min}$ at room temperature, quenched with $0.1 \mathrm{M}$ glycine for $30 \mathrm{~min}$ at room temperature, and washed with Tx buffer $(0.2 \%$ gelatin, $300 \mathrm{mM} \mathrm{NaCl}, 0.3 \%$

TritonX-100 in PBS). The sections were incubated with a mixture of rat anti-PH3 (Abcam, ab10543, 1:300), rabbit anti-Pax6 (Covance, PRB-278P, 1:200), and mouse anti-TuJ1 antibody (Covance, MMS-435P, 1:200) or rat anti-phosphohistone H3 (PH3) (Abcam, ab10543, 1:300), rabbit anti-Tbr2 (Abcam, ab23345, 1:200), and mouse anti-TuJ1 antibody (Covance, MMS-435P, 1:200) in Tx buffer for 12 hours at $4{ }^{\circ} \mathrm{C}$, and then donkey secondary antibody coupled to Alexa 488, 555 or 647 (Molecular Probes, 1:500) was incubated for 1 hour at room temperature. All sections were counterstained with Hoechst33258 (Invitrogen, 1:500). Sections were mounted in Fluoromount (Diagnostic BioSystems, CA). Confocal images were acquired using a Zeiss LSM 700 single photon point scanning confocal system using a 10x objective.

\section{Thickness of cortical wall in presumptive sulcal and non-sulcal regions}

Thickness of the cortical wall, which covered the ventricular zone (VZ) from the marginal zone (MZ), was measured in the presumptive calcarine sulcal region (the upper region of medial surface), calcarine sulcal region, and/or non-sulcal regions (the external surface) of the occipital cortex. Cortical wall thickness could be measured easily in the presumptive calcarine sulcal region in the occipital cortex, because this sulcus infolded perpendicularly to both the pial and luminal surfaces of the cortical wall. Measurements were obtained on coronal MRI images on GWs 13 to $15(\mathrm{n}=1 \mathrm{each})$ using ImageJ software (National Institutes of Health, Bethesda, USA), and on HE-sections on GW13 $(n=2)$. The mean of five points from different images/sections was used for each animal when measuring the thicknesses. oSVZ thickness was also measured at the presumptive calcarine sulcal and non-sulcal regions on 
GW 13 using HE-sections. The percentage of oSVZ thickness to the cortical wall thickness was calculated and compared between two regions. Identifications of the premature cortical layers on HE-sections were made according to the criteria of Kelava et al. (2012), as briefly summarized in Table 2.

$<<<$ Table 2 near here $>>>$

\section{D maps of cortical wall thickness in presumptive sulcal and non-sulcal regions}

We further mapped the average thickness of the cortical wall coded in color on the $3 \mathrm{D}$ surface of the cerebrum. The 3D color maps of the cortical wall thickness were obtained by computing the distance along the vertex normal for each triangle in the white matter surface to that normal's intersection with the closest vertex of a triangle on the segmented cortical surface (Zhang et al., 2003; Huang et al., 2009) by using Amira ver 5.2 (Visage Imaging, Inc., San Diego, CA, USA).

\section{Ethics}

All procedures were performed in accordance with the Laboratory Animal Welfare Act and the Guide for the Care and Use of Laboratory Animals (National Institutes of Health, Bethesda, MD, USA). All the experiments were approved by the Animal Study Committee of the Central Institute for Experimental Animals (CIEA) of Japan (Protocols 12023). 


\section{RESULTS}

\section{Cerebral sulci in adult common marmoset}

Several primary sulci were identifiable in the cerebrum of adult common marmoset by conventional ex vivo $\mathrm{T}_{2}$-weghted MRI at 7-tesla. In coronal MRI images, infolding of a lateral fissure was clearly identified on the lateral cerebral surface (Fig. 2A). A circular sulcus was distinguishable from the lateral fissure at the deep portion by slightly arching its boundary (Fig. 2A). Near the caudal end of the lateral fissure, a small indentation identified as a central sulcus was seen superiorly, and a groove of the superior temporal sulcus was observed inferiorly (Fig. 2C, C'). Interestingly, the central sulcus developed in the right hemisphere but not in the left hemisphere in this individual.

A callosal sulcus was extended rostrocaudally between the corpus callosum and overlaying the cortex of the cingulate region (Fig. $2 \mathrm{C}, \mathrm{C}$ '), and a groove of the hippocampal sulcus was definite within the hippocampal formation (Fig. 2B, C). Posteriorly to the splenium of the corpus callosum, a cingulate sulcus was identifiable as a small indentation in the cingulate region (Fig. 2D, D”).

An infolding of calcarine sulcus extended on the medial surface throughout the parietooccipital cortex (Fig. 2D, E), and at the ventral region, an occipitotemporal sulcus was present as a small indentation (Fig. 2C). More caudally, a parietooccipital sulcus was infolded in the dorsal region (Fig. 2 E, E').

$<<<$ Figure 2 near here $>>>$

In the present study, the cerebral sulci were evaluated on $\mathrm{T}_{2}$-weghted MRI scans in only 
one adult marmoset. As mentioned above, there was the viability of the presence or absence of secondary sulci, which emerged during postnatal development. As references, we evaluated incidence of the secondary sulci, i.e., the central, parietooccipital and cingulated sulci, on $\mathrm{T}_{1}$-weghted MRI scans, which had been obtained from a total 22 adult marmosets (10 males and 12 females, with ages ranging from 2.1 to 3.5 years), and the results are shown in Table 3 . Consistently, incidences of secondary sulci were variable in both adult males and females, while more than half of marmosets had all three sulci. There was no sexual difference in incidences of those three sulci. Although significant difference between the left/right side differences was not detected in the incidences of all three sulci in both sexes of marmosets, the central sulcus was slightly right-dominant in males.

$<<<$ Table 3 near here $>>>$

\section{Fetal sulcation}

The 3D volume-rendered images were calculated using MRI images, and shown in Figure 3. The rendered images reproduced the cerebral surface of the marmoset fetuses at each gestational week, and the chronology of primary sulcal emergence as defined by the rendered images well corresponded to that defined by MRI images. The cerebra of marmoset fetuses were lissencephalic till GW 11. Fetal sulcation began to indent a lateral fissure on the external surface (Figs. 3, 4), and a hippocampal sulcus on the medial surface of the hippocampus at GW 12 (Figs. 3, 5A). Accompanied by the indentation of the lateral fissure, a gap between that fissure and cranial bones was present at GW 12 (Fig. 4B). The lateral fissure infolded progressively, and became a definite groove, recognizable definitely by GW15 (Figs. 3, 4). On that gestational week, a circular sulcus was distinguishable by becoming a definite 
boundary of the lateral fissure by arching (Fig. 4A, B). Posteriorly to the lateral fissure, the superior temporal sulcus emerged as a small indentation in the temporal cortex on GW 17 (Figs. 3, 4C).

$<<<$ Figures $3 \& 4$ near here $>>>$

The hippocampal sulcus was indented on the medial surface of the hippocampus at the level of habenular nucleus on the coronal slice by GW 12 (Fig. 5A). Then, the hippocampal sulcus was located on the lateral side of the diencephalon at the level of the posterior commissar by GW 15, while descending the hippocampus posteroventrally (Fig. 5A). By GW 17, the hippocampal sulcus moved ventroanteriorly, and settled ventral of the diencephalon at the habenular nucleus level again (Fig. 5A). The 3D-rendered images facilitated the understanding of such spatiotemporal changes in the morphology of hippocampus with the location of the hippocampal sulci (Fig. 5A).

A callosal sulcus began to indent between the corpus callosum and overlying cingulate cortex on GW 15, following the emergence of the corpus callosum on GW14 (Fig. 5B). A calcarine sulcus emerged as a shallow groove in the parietooccipital cortex on GW 15 (Fig. 3; Fig. 6A), and extended caudally with progressive infolding by GW 16 (Fig. 3, 6A). Parallel to the progression of infolding of that sulcus, a vesicular lumen of the lateral ventricle in the parietooccipital cortex narrowed, and closed by GW 15 (Fig. 6A). A small indentation of occipitotemporal sulcus emerged in the ventral region of the occipital cortex, where a petrous portion of the temporal bone was contacted by protruding into an intracranial cavity on $\mathrm{GW}$ 18 (Fig. 6B). Sulcal development on PD 0 was evaluated using two marmosets. A reproducible finding regarding the cerebral sulci observable on PD 0 were obtained from MRI data of those two marmosets. All primary sulci emerged during the fetal periods were 
observed in both animals. The cingulate, central and parietooccipital sulci emerged after birth. Those sulci were observable in the marmoset cerebrum at 2 years of age, but not at least by PD 0 (Fig. 2).

$<<<$ Figures $5 \& 6$ near here $>>>$

\section{Cortical volume, cortical surface area GI, and SI}

The volume and surface area of the cerebral cortex were calculated in the marmoset fetuses at GWs 11 to 19, PD 0 and 2 years of age. The cortical volume of marmoset fetuses showed a biphasic increase: a slow phase from GWs 12 to $15\left(5.6\right.$ to $\left.301.3 \mathrm{~mm}^{3}\right)$, and a rapid phase from GW 16 to PD 0 (655.3 to $1670.0 \mathrm{~mm}^{3}$ ) (Fig. 7C). The cortical volume continuously increased after birth, reaching 2-fold the PD 0 by 2 years of age $\left(3250.0 \mathrm{~mm}^{3}\right)$ (Fig. 7C). In contrast, the cortical surface area linearly increased from GW $11\left(44.1 \mathrm{~mm}^{2}\right)$ to PD $0(1759.5$

$\mathrm{mm}^{2}$ ) (Fig. 7D). Then, the surface area continuously increased after birth, and reached 3539.7 $\mathrm{mm}^{2}$ at 2 years of age.

The developmental change in the degree of cortical convolution was evaluated based on 2D MRI slices by the GI. The mean GI exhibited a similar pattern of increase with the cortical volume during the fetal periods remaining at very low values during GWs 12 to 15 (1.006 to 1.022), and then rapidly increasing from GW 16 to PD 0 (1.067 to 1.220) (Fig. 7D). Cerebral volume continuously increased after birth (Fig. 7C), whereas the mean GI slightly decreased after birth (Fig. 7E). The mean GI marked 1.123 at 2 years of age (Fig. 7E).

The SI was calculated for quantifying the degree of cortical convolution based on 3D MRI. Like the GI, the SI increased slowly during GW 12 to 15 (0.014 to 0.039$)$, and then increased rapidly during GW 16 to PD 0 (0.119 to 0.234 ) (Fig. 7F). Then, the SI decreased from 0.234 
on PD 0 to 0.153 at 2 years of age (Fig. 7F).

$<<<$ Figure 7 near here $>>>$

Correlations of the cortical volume or surface area with two indices indicating the degree of cortical convolution, i.e., the GI and SI, were examined between GW 11 to PD 0. The cortical volume was closely correlated both with the GI $(r=0.985)$ and SI $(r=0.986)$ (Fig. $7 \mathrm{G})$. Likewise, there were markedly high correlations of the cortical surface area with the GI $(r=0.956)$ and SI $(r=0.986)$ in the cerebrum of marmoset fetuses (Fig. $7 \mathrm{H})$.

\section{Rostrocaudal GI distribution through cerebral cortex}

The rostrocaudal GI distribution in marmoset fetuses was examined for each gestational week, and the ontogeny of its patterns is shown in Figure 8. Peaks of the GI values corresponding to the lateral fissure, hippocampal sulcus, and calcarine sulcus, appeared sequentially during GWs 12 to 16 . The GI values were "1.0" in MRI slices in which those sulci were not seen. While overall GI values increased on GW 16 and thereafter, the increased GI value at the calcarine sulcus was more frequent than that at other regions. Then the GI distribution formed an adult-like distribution pattern by PD 0 (Fig. 8). The sulci emerging after birth (i.e., the cingulate, central and parietooccipital sulci) recognized as secondary sulci because of no association of those sulci with the characteristic gyrification pattern of the New World monkey group.

$<<<$ Figure 8 near here $>>>$ 


\section{Thickness of cortical wall in presumptive calcarine sulcal region}

In order to evaluate the relationship between the cortical growth and sulcal infolding, the cortical wall thickness was measured on coronal MRI images in the presumptive calcarine sulcal, calcarine sulcal, and/or non-sulcal regions on GWs 13 to 15 . Thickness of the cortical wall in the non-sulcal region was about 2-fold of that in the presumptive sulcal region during GWs 13 to 15, while the thickness in both regions progressively increased (Fig.9B).

Three-dimensional color maps of the cortical wall thicknesses were obtained from the computation of segmented cortical surfaces of presumptive calcarine sulcal, calcarine sulcal, and/or non-sulcal regions on GWs 13 to 15 (Fig. 9C). The maps revealed an expansion of cortical wall of non-sulcal region (external surface of the occipital region) with the progress of the gestational weeks. Notably, a narrow band of thin cortical wall was observed in the medial surface of occipital region, corresponding to the presumptive calcarine sulcal region on GW 13 (Fig. 9C). Then the cortical walls adjacent to that narrow band expanded, forming definite infolding of calcarine sulcus by GW 15 (Fig. 9C).

$<<<$ Figure 9 near here $>>>$

Since it is hypothesized that regional patterns of neurogenesis caused by neural progenitor cells in the SVZ help determine patterns of gyrification (Kriegstein et al. 2006; Liu et al. 2011), we measured the thickness of oSVZ in the presumptive calcarine sulcal and non-sulcal regions on GW 13 using HE-sections. Premature layers of the cortical wall in HE-sections were identified according to the criteria of Kelava et al. (2012) (see Table 2). While measurements of the cortical wall thickness by HE-sections may be slightly lower than those by MRI images, due to shrinkage of the brain tissue by dehydration by preparation of 
histological section, the cortical wall was consistently thicker in the non-sulcal region $(865.2$ $\mu \mathrm{m})$ than in the presumptive sulcal region $(384.0 \mu \mathrm{m})$ on HE-sections. Furthermore, the oSVZ thickness of the non-sulcal region was more than 2-fold of that of the presumptive sulcal region (Table 4). A percentage of oSVZ thickness relative to the cortical wall thickness was also higher in the non-sulcal region than in the presumptive sulcal region of the occipital cortex on GW 13 (Table 4).

$<<<$ Table 4 near hear $>>>$

\section{Immunohistochemistry}

Anti-Pax6 labels bRG that is densely contained in the oSVZ (Kelava et al. 2012), and anti-Tbr2 labels basal progenitors that are typically distributed in both inner SVZ (iSVZ) and oSVZ (Cheung et al., 2010). By triple-immunostaining for PH3/Pax6/Tuj1 and $\mathrm{PH} 3 / \mathrm{Tbr} / \mathrm{Tuj} 1$, both types of progenitors were present in the oSVZ in the presumptive sulcal and non-sulcal regions of the marmoset occipital cortex on GW 13 (Fig. 9D).

\section{DISCUSSION}

Though the common marmosets are generally believed to have near-lissencephalic cerebrum, the present study showed definite sulcal infolding such as the lateral fissure and calcarine sulcus on the cerebral cortical surface of the marmosets. Recently, we proposed four stages of the gyrification processes in primates based on cerebral growth and gyrification: Stage 1. Emergence of the primary sulci that demarcate cerebral lobes and gyri in limbic cortex; Stage 
2. Emergence of the primary sulci that demarcate neocortical gyri; Stage 3. Emergence of secondary and tertiary sulci; and Stage 4. Growth of sulcal length and depth (Sawada et al., 2012a). While the primary sulci do not delineate definite boundaries of structural and functional parturition (i.e., the cerebral lobes and gyri) in marmosets, the gyrification processes of this primate can be applied to our concept of gyrification stages: GWs 12 to 15 were equivalent to Stage 1; GWs 16 to 18 were equivalent to Stage 2; and Stage 3 began after birth in marmosets (Table 5). Based on the gyrification stages, the cerebral maturation in marmosets may shift later than in higher-order primates, since Stage 3 was completed by birth in humans and cynomolgus monkeys (Sawada et al., 2012a).

$<<<$ Table 5 near here $>>>$

The GI and SI are considered suitable methods for quantitatively expressing developmental trends of cortical organization (Zilles et al., 1988, 1989; Dubois et al., 2008). In the present study, the mean GI in the adult marmoset cerebrum was 1.123 , indicating the gyrencephalic brain, because it is generally regarded to be the lissencephalic brain when the mean GI = 1, but the gyrencephalic brain when the mean GI > 1 (Zilles et al., 1997). The SI in the adult marmoset cerebrum marked 0.153 , regarding the gyrencephalic brain as well.

Correlations of GI and SI to the overall growth of the brain, the volume and/or surface area of the cerebral cortex have been revealed in humans (Armstrong et al., 1991; Dubois et al., 2008) and cynomolgus monkeys (Sawada et al. 2010). Consistent with those studies, either the mean GI or SI was closely correlated with the cortical volume (GI, $r=0.986$; SI, $r=0.986)$ and the cortical surface area (GI, $r=0.956$; SI, $r=0.986$ ) of the marmoset cerebrum in the present study. Therefore, an expansion of the cerebral cortex may be involved in the cortical convolution during fetal periods in the marmosets. 
In a previous study by Kochounov et al. (2010), the average sulcal length and depth reached a respective $80 \%$ and $48 \%$ of adult values by the 24 th week of the 26.5 week-long gestational term in baboon fetuses, suggesting the possibility that the sulcal infolding proceeds after birth. However, both the mean GI and SI decreased through 2 years from birth (PD 0) in the marmoset cerebrum, although the volume and surface area of the cerebral cortex increased to about 2-fold that of birth by 2 years of age in the present study. Similarly, the mean GI reaches a peak at 37 weeks of age, and then decreases to the adult value in humans (Armstrong et al., 1995). Those findings, therefore, suggest that the cerebral cortex is expanded continuously after the completion of sulcal infolding progression at early postnatal ages.

The present study is, to our knowledge, the first to show the developmental pattern of the GI distribution of the New World monkey group. The GI distribution in the developing cerebrum of marmosets increased in a rostrocaudal gradient manner, resembling that of the Old World monkey group, cynomolgus monkeys (Sawada et al., 2010) and humans (Dubois et al., 2008). Likewise, the rostrocaudal pattern of sulcal emergence was seen in the developing cerebrum of a non-primate mammal, the ferret (Sawada and Watanabe, 2012). Such patterns of cortical maturation are very closely similar to the transverse neurogenetic gradient in rodent species (Takahashi et al., 1999; Caviness et al., 2003; Tarui et al., 2005). Those findings suggest that a rostrocaudal gradient of the neurogenetic and/or maturational processes of the cerebrum is phylogenetically highly-conserved across mammalian species. The development of cortical convolutions may track the evolutionary trajectory of the cerebral maturation across species.

Several hypotheses have been reported with regard to the gyrification mechanism, i.e., genetic control (Rakic, 2004), differential growth of inner and outer cortical strata (Richman et al., 1975), cortical growth (Toro and Burnod, 2005) and tension from white matter axons 
(van Essen, 1997; Hilgetag and Barbas, 2006; Mota and Herculano-Houzel, 2010; Sawada et al., 2011). In the present study, we found that the cortical wall in the marmoset occipital cortex was thicker in the non-sulcal region than in the presumptive sulcal region, preceding the emergence of calcarine sulcus on GW 15. This result supports the hypothesis of regional differences in the cortical growth by genetic control (gyrogenetic theory) (Welker, 1990). The bRG have been found in greater numbers in the oSVZ of the developing cortex of gyrencephalic mammals such as humans and ferrets (Hansen et al., 2010; Fietz et al., 2010; Reillo and Borrell, 2012) than in the developing cortex of lissencephalic mammals such as mice (Shitamukai et al., 2011; Wang et al., 2011). The bRG were found to be one of the responsible factors for the emergence and evolution of cortical convolutions (Nonaka-Kinoshita et al., 2013). Gyrification could be induced by a manipulation of novel nuclear antigens such Tmp1 and BAF170 by increasing the basal progenitor pool containing bRG in mice (Stahl et al., 2013; Tuoc et al., 2013). On the other hand, some researchers speculate that bRG in the oSVZ might not be necessarily correlated with gyrification and phylogeny based on the inconsistent relationship between bRG abundance in the oSVZ and gyrification, when common marmoset and agouti (a near-gyrencephalic rodent) are compared side by side (Hevner and Haydar, 2012). However, from the aspect of ontogeny, we found that the oSVZ is massively expanded in the marmoset cerebrum at embryonic days (E) 92-95 (Kelava et al. 2012) around the beginning of sulcal indentation at GW 12 (E86) in the present study. The oSVZ with Pax6-expressing bRG abundance appeared in macaques from E65 (Martínez-Cerdeño et al., 2012), immediately before the onset of sulcation in macaque (E70) (Fukunishi et al., 2006). In the present study, the oSVZ in non-sulcal regions of the marmoset fetus was shown to be thicker than that in the presumptive calcarine sulcal region on GW 13, preceding the infolding of the calcarine sulcus. Differential expansion of the oSVZ may be involved in gyral convolution and sulcal infolding in the developing cerebrum. 
The primary sulci, i.e., the lateral fissure, calcarine sulcus, and superior temporal sulcus, are phylogenetically highly conserved among primate species (Weiss and Aldridge 2003). Since the primary sulci emerge in a regular sequence during mid- to late-gestational periods in humans (Chi, 1977; Dubois et al., 2008) and cynomolgus monkeys (Sawada et al., 2012b), the extent and pattern of the sulcal development can provide clues to cerebral abnormalities. Recently, we reported the degree of calcarine sulcus infolding on GW 14.3 as a stable index for cerebral maturity (Fukunishi et al., 2011), and the primary sulcal lengths as a brief index for evaluating the region-related convolutions of the cerebral cortex in cynomolgus monkeys (Imai et al., 2011). Furthermore, gyrification abnormality is noted in human developmental and psychological disorders such as schizophrenia and autism (Kulynych et al., 1997; Levitt et al., 2003; Bonnici et al., 2007). In the present study, the chronology of primary sulcal emergences was comparable with that in higher-order primates such as cynomolgus monkeys (the Old World monkey group) and humans (summarized in Table 5). Due to its characteristic small body size of 250-400 g (Power et al. 2001), high fertility (Stevenson 1976), early sexual maturity (Hearn et al., 1978) and high cognitive functions (Epple, 1968), we concluded that the marmosets are appropriate as a non-human primate model for investigating developmental and psychological disorders with the gyrification abnormality, teratogenicity of environment factors on the cerebrum, and the gyrification mechanism.

\section{Acknowledgements}

This work was supported by grants from the "Highly creative animal model development for brain sciences" study carried out under the Strategic Research Program for Brain Sciences; and by grants from the Ministry of Education, Culture, Sports, Science and Technology (MEXT) of Japan to H.O.; from the Japan Science and Technology Corporation (JST) to H.O. 
and E.S.; and from the "Funding Program for World-leading Innovative R\&D on Science and Technology" to H.O. This work was also supported by a Keio University grant-in-aid for encouragement of young medical scientists. 


\section{REFERENCES}

Armstrong E, Curtis M, Buxhoeveden DP, Fregoe C, Zilles K, Casanova MF, McCarthy WF (1991) Cortical gyrification in the rhesus monkey: a test of the mechanical folding hypothesis. Cereb Cortex 1:426-432.

Bonnici HM, William T, Moorhead J, Stanfield AC, Harris JM, Owens DG, Johnstone EC, Lawrie SM (2007) Pre-frontal lobe gyrification index in schizophrenia, mental retardation and comorbid groups: an automated study. Neuroimage 35:648-654.

Caviness VS Jr, Goto T, Tarui T, Takahashi T, Bhide PG, Nowakowski RS (2003) Cell output, cell cycle duration and neuronal specification: a model of integrated mechanisms of the neocortical proliferative process. Cereb Cortex 13:592-598.

Cheung AF, Kondo S, Abdel-Mannan O, Chodroff RA, Sirey TM, Bluy LE, Webber N, DeProto J, Karlen SJ, Krubitzer L, Stolp HB, Saunders NR, Molnár Z (2010) The subventricular zone is the developmental milestone of a 6-layered neocortex: comparisons in metatherian and eutherian mammals. Cereb Cortex 20:1071-1081.

Chi JG, Dooling EC, Gilles FH. (1977) Gyral development of the human brain. Ann Neurol $1: 86-93$.

Dubois J, Benders M, Cachia A, Lazeyras F, Leuchter RHV, Sizonenko SV, Borradori-Tolsa C, Mangin JF, Hüppi PS (2008) Mapping the early cortical folding process in the preterm newborn brain. Cereb Cortex 18:1444-1454.

Epple G (1968) Comparative studies on vocalization in marmoset monkeys (Hapalidae). Folia Primatol (Basel) 8:1-40.

Fietz SA, Kelava I, Vogt J, Wilsch-Bräuninger M, Stenzel D, Fish JL, Corbeil D, Riehn A, Distler W, Nitsch R, Huttner WB (2010) OSVZ progenitors of human and ferret neocortex are epithelial-like and expand by integrin signaling. Nat Neurosci 13:690-699. 
Fujiyoshi K, Yamada M, Nakamura M, Yamane J, Katoh H, Kitamura K, Kawai K, Okada S, Momoshima S, Toyama Y, Okano H (2007) In vivo tracing of neural tracts in the intact and injured spinal cord of marmosets by diffusion tensor tractography. J Neurosci 27:11991-11998.

Fukunishi K, Sawada K, Kashima M, Sakata-Haga H, Fukuzaki K, Fukui Y. (2006) Development of cerebral sulci and gyri in fetuses of cynomolgus monkeys (Macaca fascicularis). Anat Embryol (Berl) 211:757-764.

Fukunishi K, Sawada K, Kashima M, Saito S, Sakata-Haga H, Sukamoto T, Aoki I, Fukui Y (2011) Correlation between formation of the calcarine sulcus and morphological maturation of the lateral ventricle in cynomolgus monkey fetuses. Acta Neurobiol Exp (Wars) 71:381-386.

Hansen DV, Lui JH, Parker PRL, Kriegstein AR. (2010) Neurogenic radial glia in the outer subventricular zone of human neocortex. Nature 464:554-561.

Hearn JP, Abbott DH, Chambers PC, Hodges JK, Lunn SF (1978) Use of the common marmoset, Callithrix jacchus, in reproductive research. Primates Med 10:40-49.

Herculano-Houzel S, Mota B, Wong P, Kaas JH. (2010) Connectivity-driven white matter scaling and folding in primate cerebral cortex. Proc Natl Acad Sci U S A 107:19008-19013.

Hevner RF, Haydar TF (2012) The (not necessarily) convoluted role of basal radial glia in cortical neurogenesis. Cereb Cortex 22:465-468.

Hikishima K, Sawada K, Murayama A, Komaki Y, Kawai K, Sato N, Inoue T, Itoh T, Momoshima S, Iriki A, Okano HJ, Sasaki E, Okano H (2013) Atlas of the developing brain of the marmoset monkey using magnetic resonance histology. Neuroscience 230: $102-113$.

Hilgetag CC, Barbas AH (2006) Role of mechanical factors in the morphology of the primate 
cerebral cortex. PLoS Comput Biol 2:e22.

Huang H, Xue R, Zhang J, Ren T, Richards LJ, Yarowsky P, Miller MI, Mori S (2009) Anatomical characterization of human fetal brain development with diffusion tensor magnetic resonance imaging. J Neurosci 29: 4263-4273.

Imai N, Sawada K, Fukunishi K, Sakata-Haga H, Fukui Y (2011) Sexual dimorphism of sulcal length asymmetry in cerebrum of adult cynomolgus monkeys (Macaca fascicularis). Congenit Anom (Kyoto) 51:161-166.

Iwanami A, Yamane J, Katoh H, Nakamura M, Momoshima S, Ishii H, Tanioka Y, Tamaoki N, Nomura T, Toyama Y, Okano H (2005a) Establishment of graded spinal cord injury model in a non-human primate: the common marmoset. J Neurosci Res 80:172-181. Iwanami A, Kaneko S, Nakamura M., Kanemura Y, Mori H, Kobayashi S, Yamasaki M, Momoshima S, Ishii H, Ando K, Tanioka Y, Tamaoki N, Nomura T, Toyama Y, Okano H (2005b) Transplantation of human neural stem cells for spinal cord injury in primates. J Neurosci Res 80:182-190.

Kashima M, Sawada K, Fukunishi K, Sakata-Haga H, Tokado H, Fukui Y (2008) Development of cerebral sulci and gyri in fetuses of cynomolgus monkeys (Macaca fascicularis). II. Gross observation of the medial surface. Brain Struct Funct 212:513-520.

Kelava I, Reillo I, Murayama AY, Kalinka AT, Stenzel D, Tomancak P, Matsuzaki F, Lebrand C, Sasaki E, Schwamborn JC, Okano H, Huttner WB, Borrell V. (2012) Abundant occurrence of basal radial glia in the subventricular zone of embryonic neocortex of a lissencephalic primate, the common marmoset Callithrix jacchus. Cereb Cortex $22: 469-481$.

Kobayashi Y, Okada Y, Itakura G, Iwai H, Nishimura S, Nori S, Hikishima K, Konomi T, Fujiyoshi K, Tsuji O, Toyama Y, Yamanaka S, Nakamura M, Okano H (2012) Human 
iPSC-derived neural stem cells promote functional recovery after spinal cord injury in common marmosets without tumorigenicity. PLoS ONE 7:e52787.

Kochounov P, Glahn DC, Fox P, Lancaster JL, Saleem K, Shelledy W, Zilles K, Thompson PM, Coulon O, Mangin JF, Blangero J, Rogers J. (2010) Genetics of primary cerebral gyrification: heritability of length, depth and area of primary sulci in an extended pedigree of baboons. Neuroimage 53:1126-1134.

Kriegstein A, Noctor S, Martínez-Cerdeño V (2006) Patterns of neural stem and progenitor cell division may underlie evolutionary cortical expansion. Nat Rev Neurosci 7:883-890.

Kulynych JJ, Luevano LF, Jones DW, Weinberger DR (1997) Cortical abnormality in schizophrenia: an in vivo application of the gyrification index. Biol Psychiatry 41:995-999.

Levitt JG, Blanton RE, Smalley S, Thompson PM, Guthrie D, McCracken JT, Sadoun T, Heinichen L, Toga AW (2003) Cortical sulcal maps in autism. Cereb Cortex 13:728-735.

Lui JH, Hansen DV, Kriegstein AR (2011) Development and evolution of the human neocortex. Cell 146:18-36.

Martínez-Cerdeño V, Cunningham CL, Camacho J, Antczak JL, Prakash AN, Cziep ME, Walker AI, Noctor SC (2012) Comparative analysis of the subventricular zone in rat, ferret and macaque: evidence for an outer subventricular zone in rodents. PLoS One 7:e30178.

Maudgil DD, Free SL, Sisodiya SM, Lemieux L, Woermann FG, Fish DR, Shorvon SD (1988) Identifying homologous anatomical landmarks on reconstructed magnetic resonance images of the human cerebral cortical surface. J Anat 193:559-571.

Naidich TP, Grant JL, Altman N, Zimmerman RA, Birchansky SB, Braffman B, Daniel JL (1994) The developing cerebral surface. Preliminary report on the patterns of sulcal and gyral maturation — anatomy, ultrasound, and magnetic resonance imaging. Neuroimaging 
Clin North Am 4:201-240.

Newman JD, Kenkel WM, Aronoff EC, Bock NA, Zametkin MR, Silva AC (2009) A combined histological and MRI brain atlas of the common marmoset monkey, Callithrix jacchus. Brain Res Rev 62:1-18.

Nonaka-Kinoshita M, Reillo I, Artegiani B, Martínez-Martínez MÁ, Nelson M, Borrell V, Calegari F (2013) Regulation of cerebral cortex size and folding by expansion of basal progenitors. EMBO J 32: 1817-1828.

Power RA, Power, ML, Layne, DG, Jaquish CE, Oftedal OT, Tardif SD (2001) Relations among measures of body composition, age, and sex in the common marmoset monkey (Callithrix jacchus). Comp Med 51:218-223.

Prayer D, Kasprian G, Krampl E, Ulm B, Witzani L, Prayer L, Brugger PC (2006) MRI of normal fetal brain development. Eur J Radiol 57:199-216.

Rakic P (2004) Neuroscience. Genetic control of cortical convolutions. Science 303:1983-1984.

Reillo I, Borrell V (2012). Germinal zones in the developing cerebral cortex of ferret: ontogeny, cell cycle kinetics, and diversity of progenitors. Cereb Cortex 22, 2039-2054.

Richman DP, Stewart RM, Hutchinson JW, Caviness VS Jr (1975) Mechanical model of brain convolutional development. Science 189:18-21.

Sawada K, Fukunishi K, Kashima M, Saito S, Aoki I, Fukui Y (2012a) Fetal gyrification in cynomolgus monkeys: a concept of developmental stages of gyrification. Anat Rec (Hoboken) 295:1065-1074.

Sawada K, Fukunishi K, Kashima M, Imai N, Saito S, Sakata-Haga H, Aoki I, Fukui Y (2012b) Neuroanatomic and MRI references for normal development of cerebral sulci of laboratory primate, cynomolgus monkeys (Macaca fascicularis). Congenit Anom (Kyoto) $52: 16-27$. 
Sawada K, Sun X-Z, Fukunishi K, Kashima M, Satio S, Aoki I, Sakata-Haga H, Fukui Y (2011) Development of corticocortical long associative fibers in cynomolgus monkey fetal cerebrum analyzed using DTI: its relation to sulcal formation. Adv Studies Biol $3: 133-150$.

Sawada K, Sun XZ, Fukunishi K, Kashima M, Sakata-Haga H, Tokado H, Aoki I, Fukui Y (2009) Developments of sulcal pattern and subcortical structures of the forebrain in cynomolgus monkey fetuses: 7-tesla magnetic resonance imaging provides high reproducibility of gross structural changes. Brain Struct Funct 213:469-480.

Sawada K, Sun X-Z, Fukunishi K, Kashima M, Satio S, Sakata-Haga H, Sukamoto T, Aoki, I, Fukui Y (2010) Ontogenetic pattern of gyrification in fetuses of cynomolgus monkeys Neuroscience 167:735-740.

Sawada K, Watanabe M (2012) Development of cerebral sulci and gyri in ferrets (Mustela putorius). Congenit Anom (Kyoto) 52:168-175.

Shitamukai A, Konno D, Matsuzaki F (2011) Oblique radial glial divisions in the developing mouse neocortex induce self-renewing progenitors outside the germinal zone that resemble primate outer subventricular zone progenitors. J Neurosci 31:3683-3695.

Stahl R, Walcher T, De Juan Romero C, Pilz GA, Cappello S, Irmler M, Sanz-Aquela JM, Beckers J, Blum R, Borrell V, Götz M (2013) Trnp1 regulates expansion and folding of the mammalian cerebral cortex by control of radial glial fate. Cell 153: 535-549.

Stevenson MF (1976) Birth and perinatal behaviour in family groups of the common marmoset (Callithrix jacchus jacchus), compared to other primates. J Hum Evol $5: 365-381$.

Takahashi T, Goto T, Miyama S, Nowakowski RS, Caviness VS Jr (1999) Sequence of neuron origin and neocortical laminar fate: relation to cell cycle of origin in the developing murine cerebral wall. J Neurosci 19:10357-10371. 
Tarui T, Takahashi T, Nowakowski RS, Hayes NL, Bhide PG, Caviness VS (2005)

Overexpression of p27 Kip 1, probability of cell cycle exit, and laminar destination of neocortical neurons. Cereb Cortex 15:1343-1355.

Toro R, Burnod Y (2005) A morphogenetic model for the development of cortical convolutions. Cereb Cortex 15:1900-1913.

Tuoc TC, Boretius S, Sansom SN, Pitulescu ME, Frahm J, Livesey FJ, Stoykova A (2013) Chromatin regulation by BAF170 controls cerebral cortical size and thickness. Dev Cell 25: 256-269.

van Essen DC (1997) A tension-based theory of morphogenesis and compact wiring in the central nervous system. Nature 385:313-318.

Wang X, Tsai JW, LaMonica B, Kriegstein AR (2011) A new subtype of progenitor cell in the mouse embryonic neocortex. Nat Neurosci 14:555-561.

Weiss K, Aldridge K (2003) What stamps the wrinkle on the brow. Evol Anthropol $12: 205-210$.

Welker W. Why does cerebral cortex fissure and fold? A review of determinations of gyri and sulci. In: Comparative structure and evolution of cerebral cortex, Part II. 1990; vol 8B. Plenum, New York, p 3-136.

Yamada M, Momoshima S, Masutani Y, Fujiyoshi K, Abe O, Nakamura M, Aoki S, Tamaoki N, Okano H (2008) Diffusion-tensor neuronal fiber tractography and manganese-enhanced MR imaging of primate visual pathway in the common marmoset: preliminary results. Radiology 249:855-864.

Yuasa S, Nakamura K, Kohsaka S (2010) Stereotaxic atlas of the marmoset brain. volume 1. Nissl-stained sections and their diagrams. Japan: National Institute of Neuroscience, National Center of Neurology and Psychiatry.

Zhang J, Richards LJ, Yarowsky P, Huang H, van Zijl PC, Mori S (2003) Three-dimensional 
anatomical characterization of the developing mouse brain by diffusion tensor microimaging. Neuroimage 20:1639-1648.

Zilles K, Armstrong E, Moser KH, Schleicher A, Stephan H. (1989) Gyrification in the cerebral cortex of primates. Brain Behav Evol 34:143-150.

Zilles K, Armstrong E, Schleicher A, Kretschmann HJ (1988) The human pattern of gyrification in the cerebral cortex. Anat Embryol (Berl) 179:173-179.

Zilles K, Schleicher A, Langemann C, Amunts K, Morosan P, Palomero-Gallagher N, Schormann T, Mohlberg H, Bürgel U, Steinmetz H, Schlaug G, Roland PE (1997) Quantitative analysis of sulci in the human cerebral cortex: development, regional heterogeneity, gender difference, asymmetry, intersubject variability and cortical architecture. Hum Brain Mapp 5:218-221. 


\section{FIGURE LEGENDS:}

Fig. 1 Identification of cerebral sulci. (A) Attempted curvature analysis of digital cortical surface. Small indentations indicating secondary sulci and small indentations caused by surface blood vessels are indistinguishable in this analysis. (B) Visual identification of cerebral sulci in MRI slices. Arrowheads indicate indentations on the cerebral surface corresponding to the central sulcus, and parietooccipital sulcus, and also indentations caused by a blood vessel. Dotted lines in the lower three images indicate the boundaries between the gray and white matter. Such boundaries below the central and parietooccipital sulci are curved slightly, but the boundary below the blood vessel indentation is not. Therefore, small indentations of the cerebral sulci can be defined using the criteria, "a clear indentation at the cerebral surface with curvature of the adjacent subcortical white matter." BV: indentation of blood vessel on cerebral cortical surface, lf: lateral fissure, pos: parietooccipital sulcus.

Fig. 2 Coronal $\mathrm{T}_{2}$-weighted MRI images of cerebrum of adult (2-year-old) common marmoset. MRI images in (A) to (E) are at the levels indicated in lateral image of left hemisphere (F). In the adult marmoset cerebrum, the lateral fissure (lf) (A, B), occipitotemporal sulcus (ots) (D), and calcarine sulcus (D, E) were observable on the cerebral surface. At the deep portion of the lateral fissure, the circular sulcus (cirs) was distinguishable by slightly arching its boundary (A). Boxed areas in (B) to (C) are shown at magnification of the cerebral surface, with indentations of the central sulcus (cs) (B'), superior temporal sulcus (sts) (C'), callosal sulcus (cas) (C"), cingulate sulcus (cgs) (D”) and parietooccipital sulcus (pos) (E'), respectively. cc, corpus callosum.

Fig. 3 3D volume-rendered images of the cerebrum of common marmoset on gestational weeks 11 to 19 . Rendered images reproduced the cerebral surface of the marmoset fetuses at 
each gestational week, and the chronology of primary sulcal emergence as defined by the rendered images well corresponded to that defined by MRI images. If, lateral fissure; cal, calcarine sulcus; cas, callosal sulcus; ots, occipitotemporal sulcus; sts, superior temporal sulcus.

Fig. 4 Axial and coronal MRI images indicated the developments of lateral fissure, circular sulcus and superior temporal sulcus. (A) Development of lateral fissure (lf) (low magnification images). Axial MRI images of the left hemisphere at the anterior commissure from GWs 12 to 18 are shown. (B) Development of lateral fissure (high magnification images). Axial MRI images of the lateral fissure and circular sulcus from GWs 12 to 18 are shown. Lateral fissure was first seen on GW 12, but not yet on GW 11. Circular sulcus (cirs) was distinguishable by becoming a definite boundary to the lateral fissure by arching. Asterisks $(*)$ indicate intracranial space between the lateral fissure and cranial bones. Gap was filled with the cortex adjacent to the lateral fissure by GW18. (C) Development of superior temporal sulcus (sts). Superior temporal sulcus emerged as a small indentation on GW 17 shown at a high magnification (see inset).

Fig. 5 Coronal MRI images indicated the developments of hippocampal and callosal sulci. (A) Development of hippocampal sulcus (his). Upper and lower rows of MRI images are at the levels indicated in the 3D volume-rendered images of medial cerebral surface (middle rows). Rostral level of MRI images included the habenular nucleus in the diencephalon, and the caudal level of MRI images included the posterior commissure in the cerebrum. On the rendered images, the hippocampus and hippocampal sulcus (his) coded in yellow and red colors, respectively. Hippocampal sulcus was located on the lateral side of the diencephalon at the level of the posterior commissar by GW 15, descended posteroventrally, and settled 
ventral of the diencephalon at the habenular nucleus level by GW 17. (B) Development of callosal sulcus (cas). The callosal sulcus began to indent between the corpus callosum (cc) and overlying cingulate cortex by GW 15, following emergence of the corpus callosum on GW14.

Fig. 6 Coronal MRI images indicated the developments of calcarine and occipitotemporal sulcus. (A) Development of calcarine sulcus (cal). MRI images indicate from GWs 15 to 16 are at the levels indicated in lateral image of left hemisphere. Calcarine sulcus emerged in the parietooccipital cortex lateral to the superior colliculus (sc) on GW 15, and extended caudally with progressive infolding by GW 16 . Asterisks $\left(^{*}\right)$ indicate vesicular lumen of lateral ventricle in the occipital cortex on GW 14 that narrowed, and closed by GW 15. (B) Development of occipitotemporal sulcus (ots). Boxed areas indicate high magnification in respective right images. Occipitotemporal sulcus (ots) emerged in the ventral region of the occipital cortex, where a petrous portion of the temporal bone $(\mathrm{Pe})$ was contacted by protruding into an intracranial cavity on GW 18.

Fig. 7 Developmental changes in cortical volume, cortical surface area, gyrification index, sulcation index, and their correlations in common marmosets from GWs 11 to PD 0. (A) Procedure and formula for calculation of gyrification index (GI). GI was determined using all MR images at the coronal plane, according to the procedure by Zilles et al. $(1988,1989)$. Outer contour represented the most superficial region of the cortex surrounding the external gyral surfaces, while excluding the inner sulci (solid line). Inner contour formed an adjacent boundary of the outer contour in addition to the sulcal groove (broken line). Measurements were obtained by manually tracing the outer and inner contours on the coronal MRI images using Scion Image software (Scion Corporation, Frederick, MD, USA). GI values were 
calculated by the ratio between the external and inner contours of each MRI image. (B)

Procedure and formula for calculation of sulcation index (SI). SI was calculated by a modified procedure originally proposed by Dubois et al. (2008). Closed surface area of the cortex, which corresponded to a smooth envelope of the external gyral surface (left image), was computed from 3D MRI images using SliceOmatic software. Surface areas of all sulcal grooves (right image) were also calculated. SI was calculated by the ratio between the sulcal surface areas and the closed cortical surface area. (C) Cortical volume. Cortical volume showed a biphasic increase: a slow phase from GWs 12 to 15 , and a rapid phase from GW 16 to PD 0. After birth, the cortical volume increased continuously, and was marked about twice of the volume at PD 0 by 2 years of age. (D) Cortical surface area. Surface area linearly increased from GW 11 to PD 0. (E) Gyrification index (GI). GI showed a biphasic increase: a slow phase from GWs 12 to 15 , and a rapid phase from GW 16 to PD 0 . The mean GI reached a plateau by PD 0. (F) Sulcation index (SI). SI showed two rapid increasing phases: from GWs 15 to 17; and from GWs 18 to 19. (G) Correlations of the cortical volume with the GI or SI. Cortical volume was closely correlated both with the GI $(r=0.985)$ and SI $(r=0.986)$. $(H)$ Correlations of the surface area with the GI or SI. Surface area was closely correlated both with the GI $(r=0.956)$ and SI $(r=0.986)$.

Fig. 8 Representative pattern of the GI distribution along its rostrocaudal course through the cerebrum of common marmosets from gestational week 11 to adult. Approximate extent of primary sulci is indicated. Peaks of GI values corresponding to the lateral fissure (lf), hippocampal sulcus (his), and calcarine sulcus (cal), appeared sequentially during GWs12 to 16. GI distribution formed an adult-like distribution pattern until PD 0 by overall increase of GI values. cas, callosal sulcus; ots, occipitotemporal sulcus; sts, superior temporal sulcus. 
Fig. 9 Changes in cortical wall thickness in presumptive calcarine sulcal and non-sulcal regions of occipital cortex of common marmosets from gestational weeks 13 to 15 . (A) Coronal MRI images of occipital cortex. Presumptive calcarine sulcal region (the upper part of medial surface; indicated by red arrowheads) was thinner than non-sulcal region (the external surfaces; indicated by blue arrowheads) on gestational weeks (GWs) 13 to 14 . Calcarine sulcus was clearly observed in the medial surface on GW 15 (orange arrowhead). (B) MRI-based measurements of thickness of cortical wall in the presumptive calcarine sulcal and non-sulcal regions from GWs $13(\mathrm{n}=1), 14(\mathrm{n}=1)$ and $15(\mathrm{n}=1)$. While thickness of the cortical wall in both regions increased progressively, about 2-fold of the cortical wall thickness was marked in the non-sulcal region when compared to the presumptive sulcal region. (C) Three-dimensional color maps of the cortical wall thicknesses obtained from the computation of segmented cortical surfaces of presumptive calcarine sulcal, calcarine sulcal, and/or non-sulcal regions on GWs $13(\mathrm{n}=1), 14(\mathrm{n}=1)$ and $15(\mathrm{n}=1)$. Compressed color scales represent ranges of the thickness: 0 to $2.0 \mathrm{~mm}$ in left 6 images, and 0 to $1.2 \mathrm{~mm}$ (low range) in a high magnification image at the right image. Arrowheads indicate the presumptive calcarine sulcal and/or calcarine sulcal region. The high magnification image revealed a narrow band of thin cortical wall, corresponding to the presumptive calcarine sulcal region on GW 13. (D) Histological profiles of the cortical wall in the presumptive sulcal and non-sulcal regions. HE-stained and immunostained sections of the presumptive sulcal and non-sulcal regions made are shown in red and blue boxed areas on MRI images. Premature layers of the cortical wall in HE-sections were identified according to the criteria of Kelava et al. (2012) (see Table 2). Triple immunostaining for PH3 (green)/Pax6 (magenta)/Tuj1 (cyan) or PH3 (green)/Tbr2 (magenta)/Tuj1 (cyan) with Hoechist staining (blue) revealed the presence of two types of progenitors, i.e., Pax6-expressing basal radial glia (bRG) and Tbr2-expressing basal progenitors in the outer subventricuolar zone (oSVZ). CP, cortical plate; iSVZ, inner 
subventricular zone; IZ, intermediate zone; MZ, marginal zone; SP, subplate; VZ, ventricular zone. 
Figure 1

\section{A. 3D curvature maps}
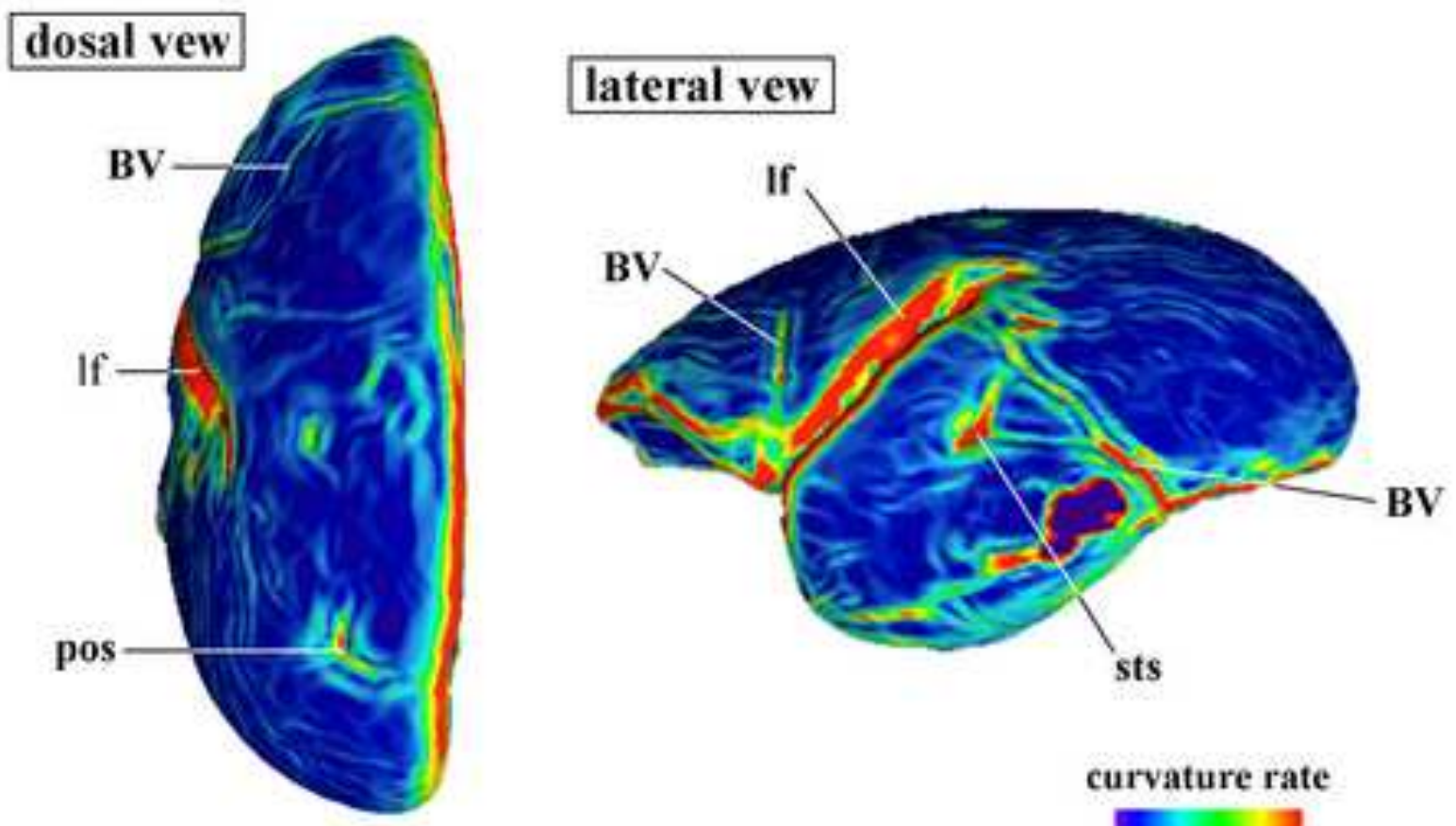

B. Curvatures of subcortical white matter
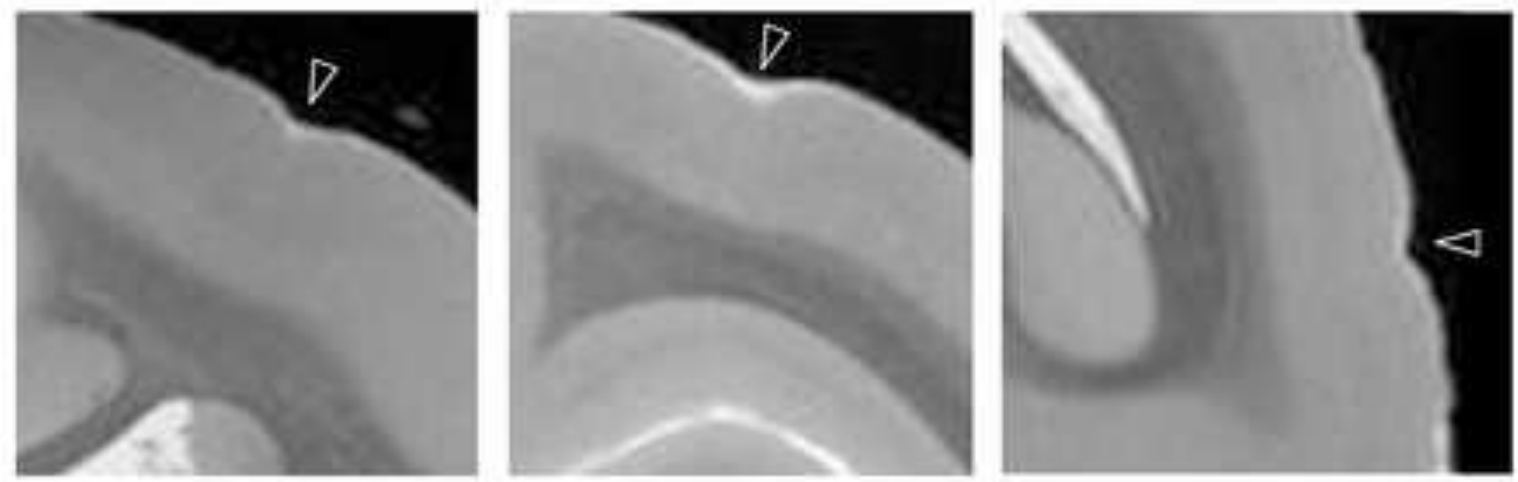

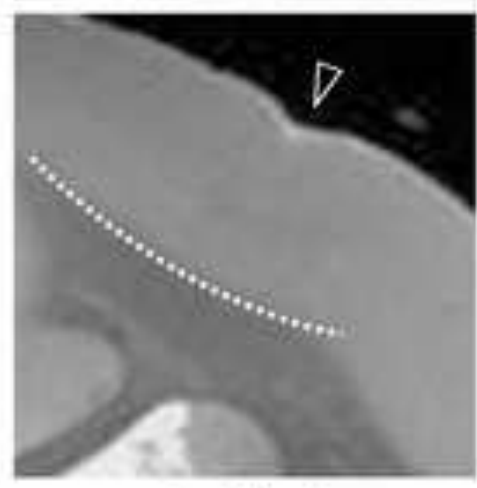

central sulcus

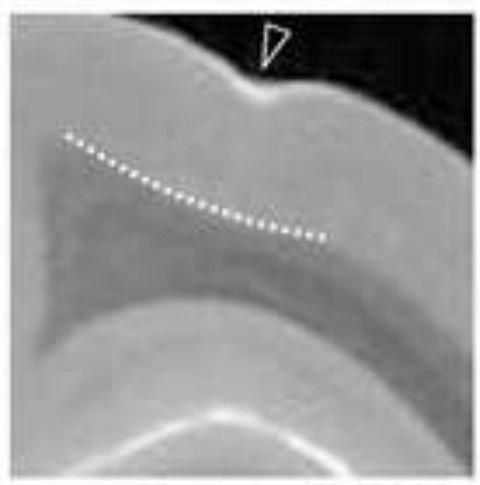

parietooccipital sulcus

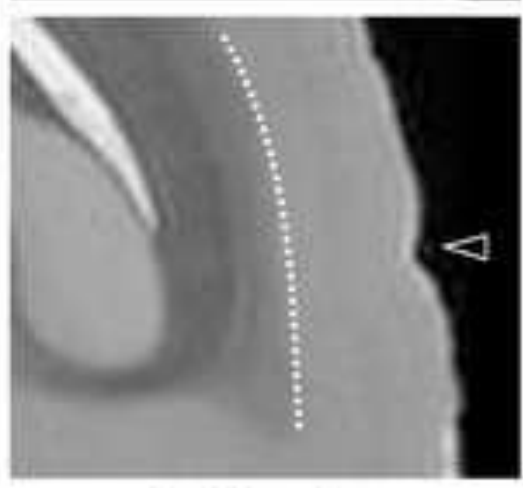

hollow by blood vessel 

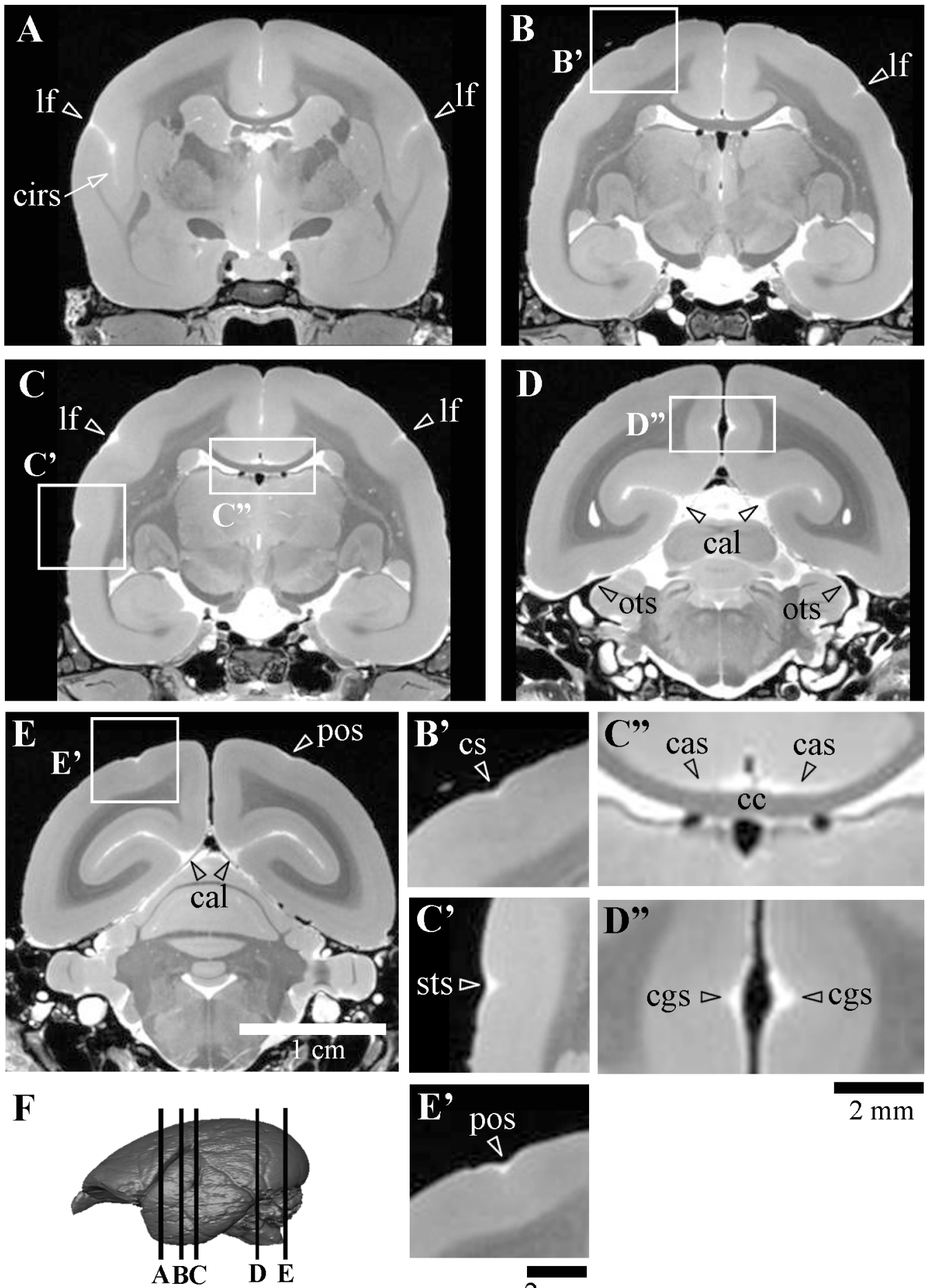

FIG 2

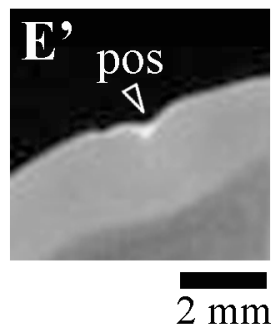




\section{GW11 GW 12 GW 13 \\ GW 14}

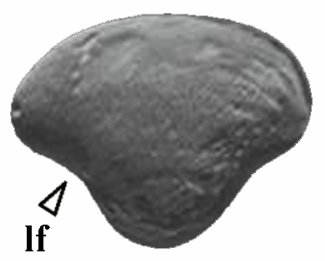

Lateral views

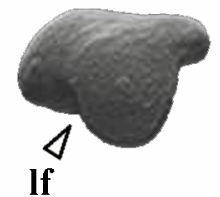

arivily

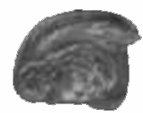

Medial views

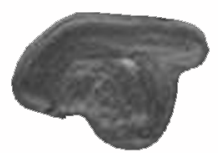

GW 17

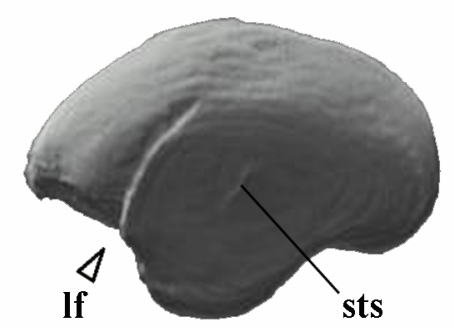

Lateral views

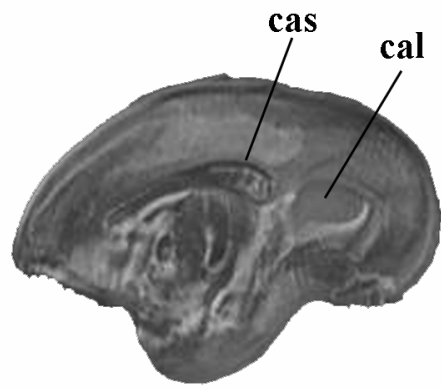

Medial views
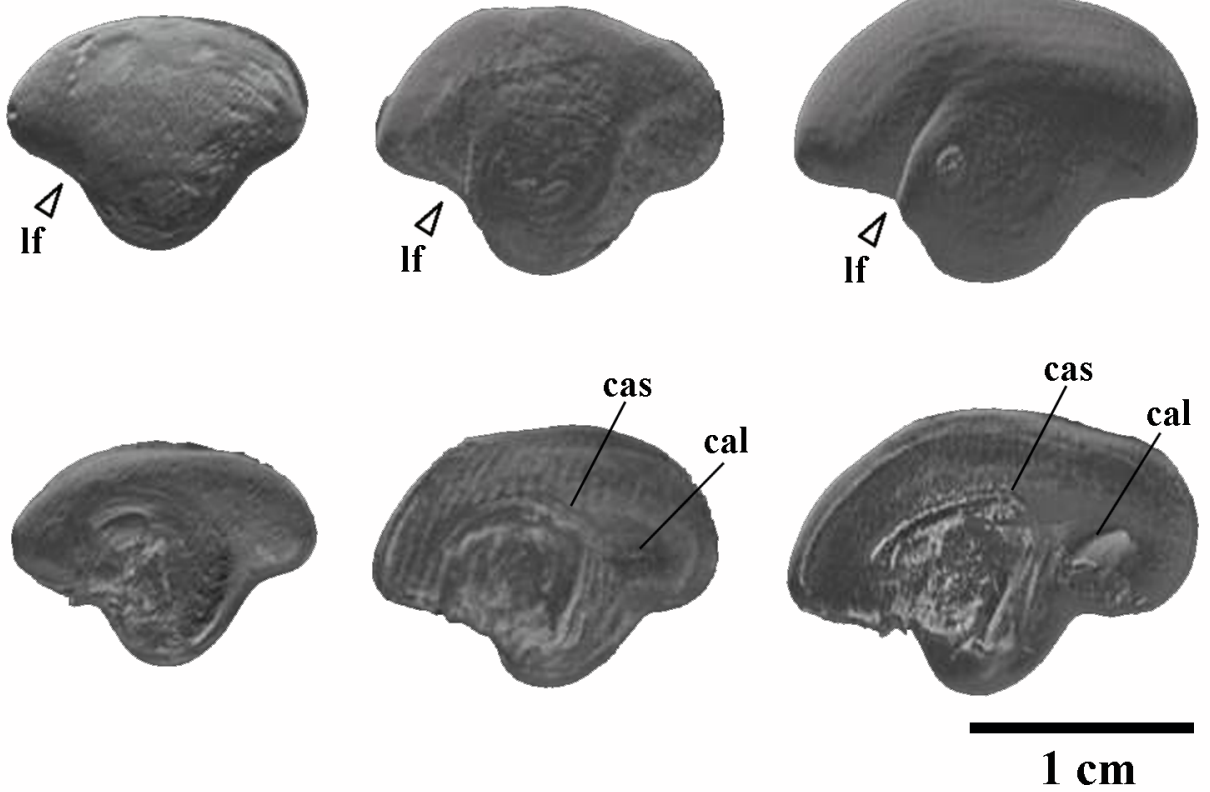

GW 18
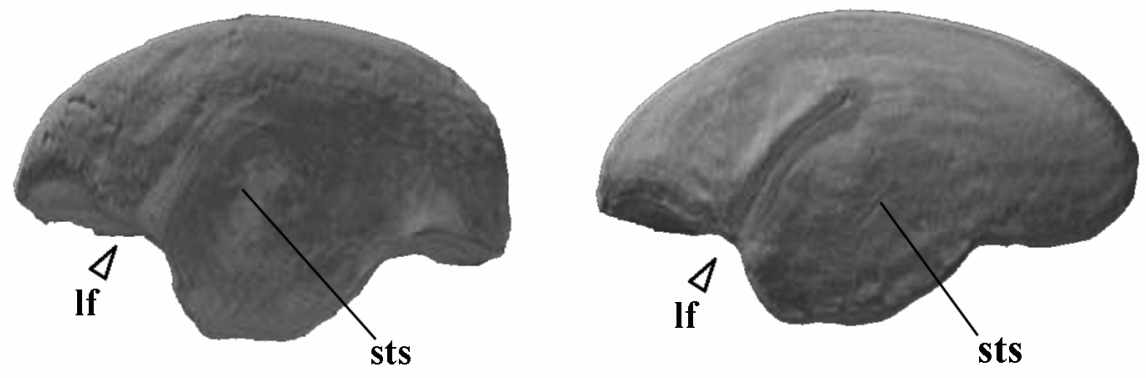

GW 19
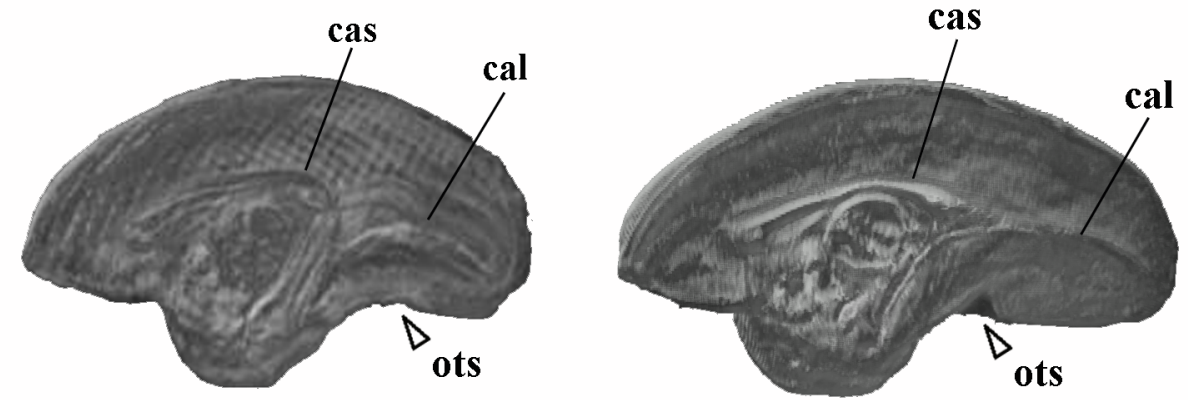
A. Development of lateral fissure (low-magnification)
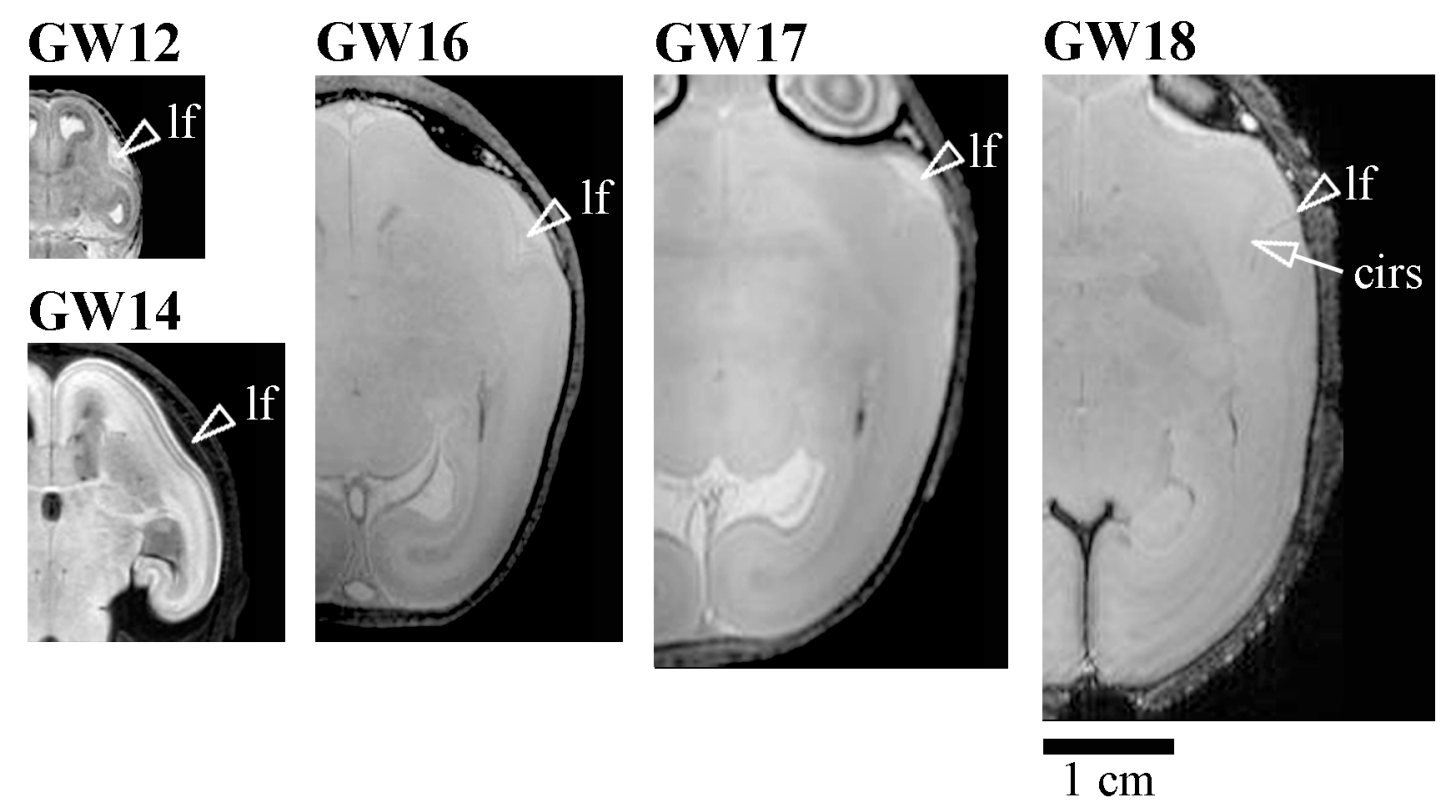

B. Development of lateral fissure (high-magnification)

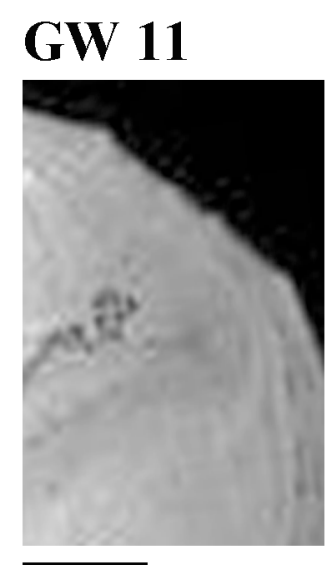

$0.5 \mathrm{~mm}$
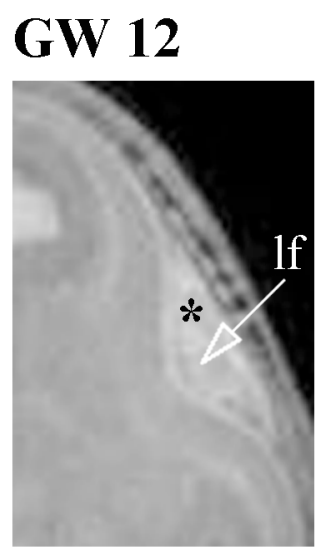

$2 \mathrm{~mm}$
GW17

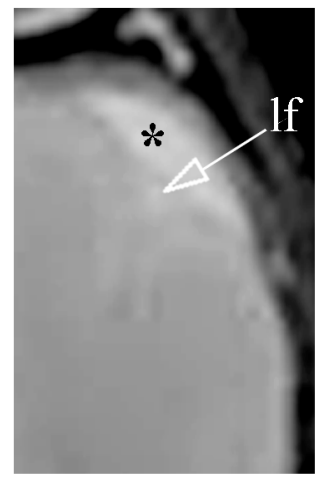

$2 \mathrm{~mm}$
GW 18

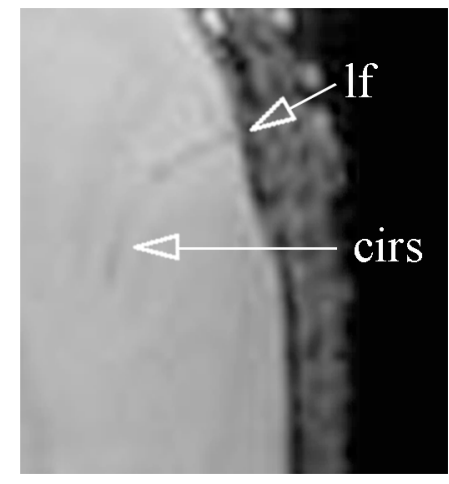

$5 \mathrm{~mm}$

C. Development of superior temporal sulcus

GW 16

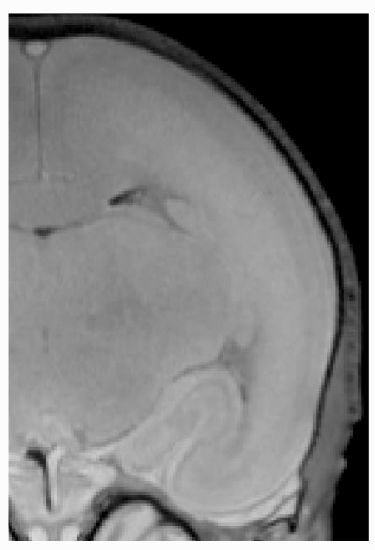

$5 \mathrm{~mm}$
GW 17

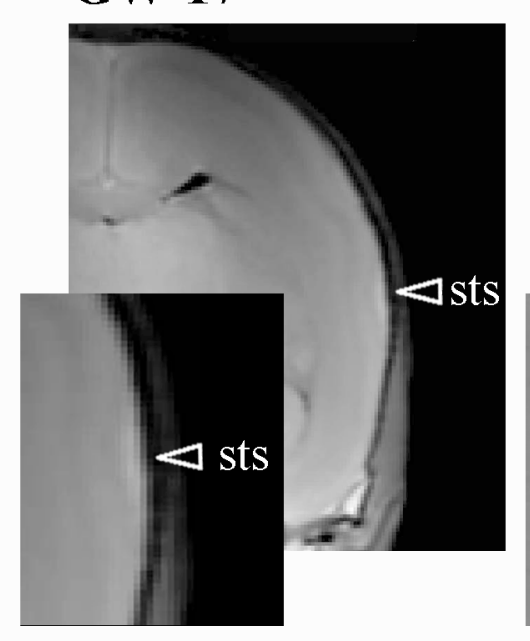

GW 18

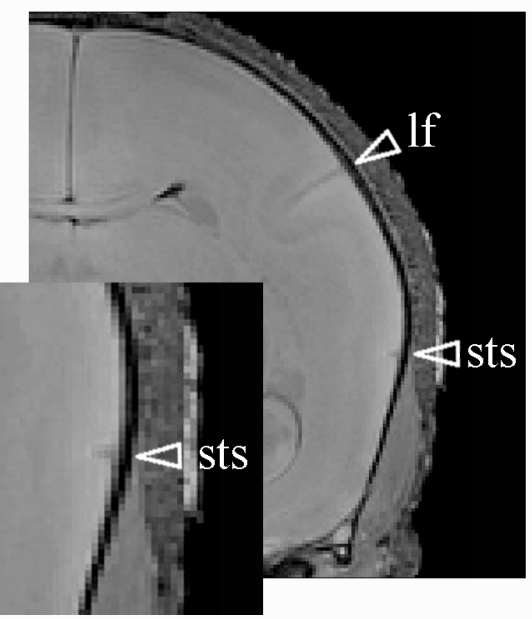




\section{A. Development of hippocampal sulcus}

\section{GW12 GW13 \\ GW15}

level of habenular nuclei
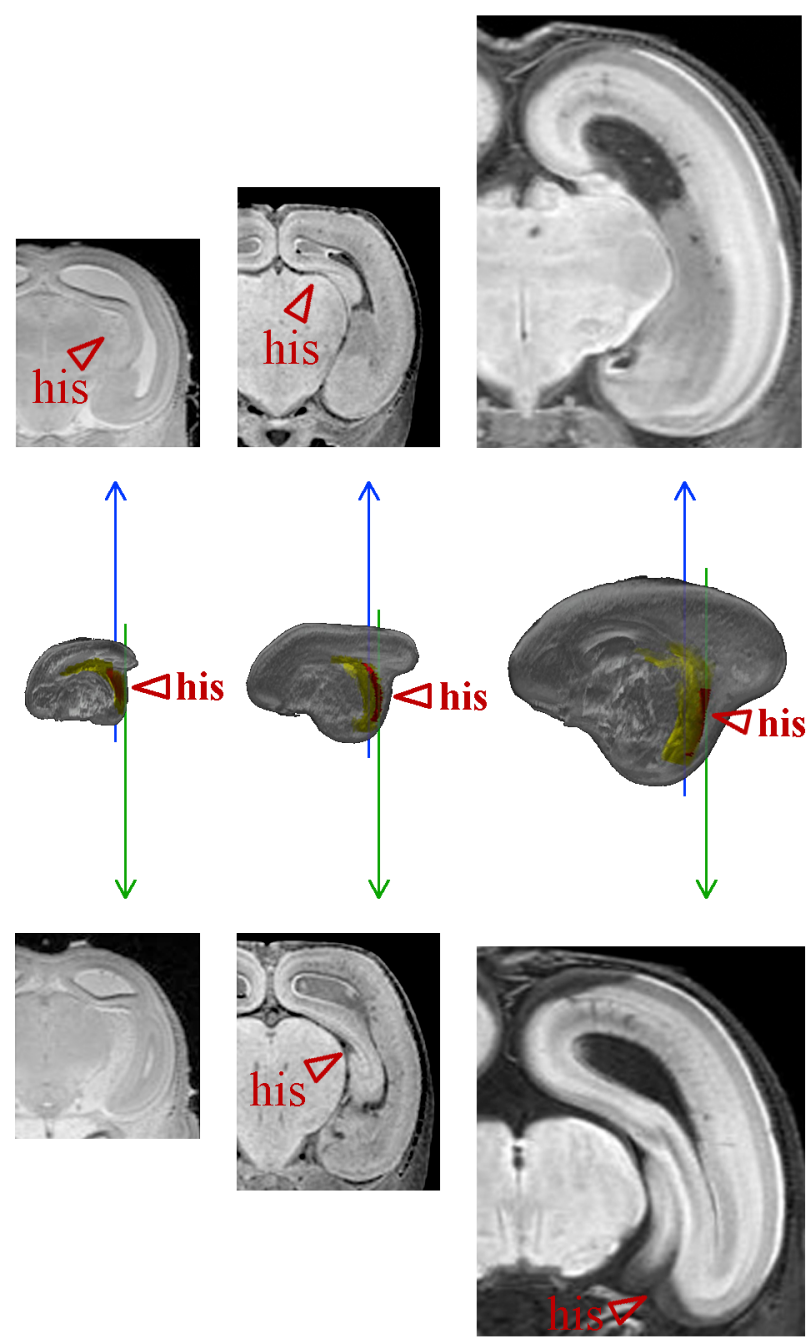

level of posterior commissure
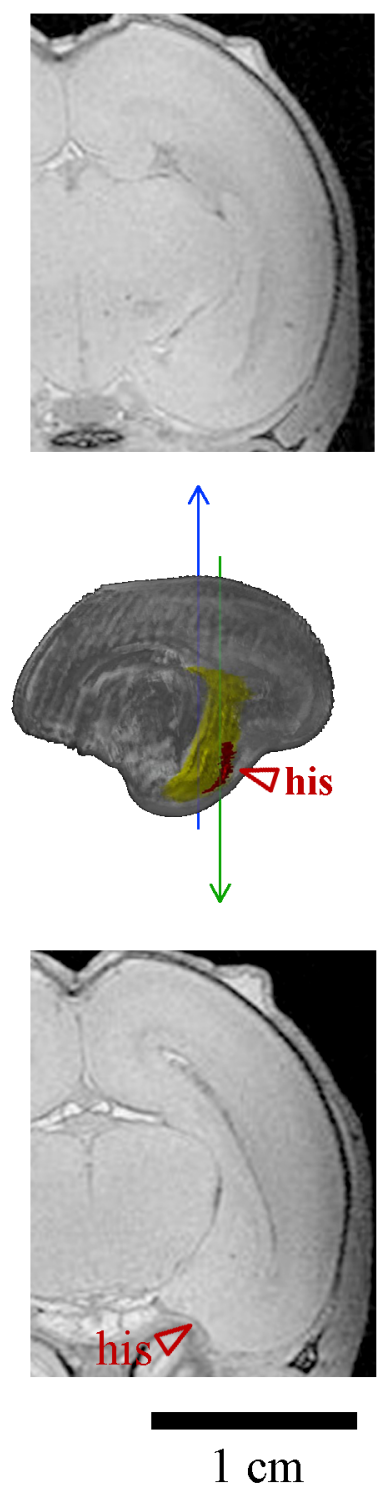

GW17
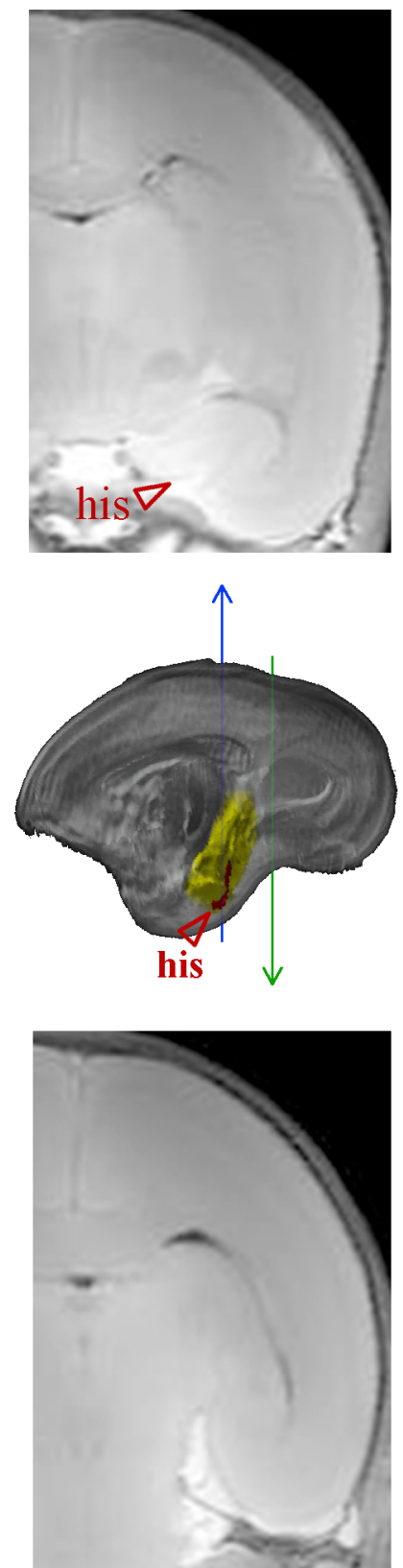

\section{B. Development of callosal sulcus}

GW 13

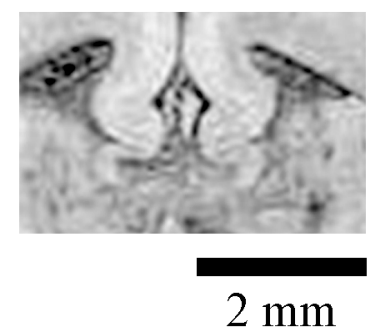

GW 14

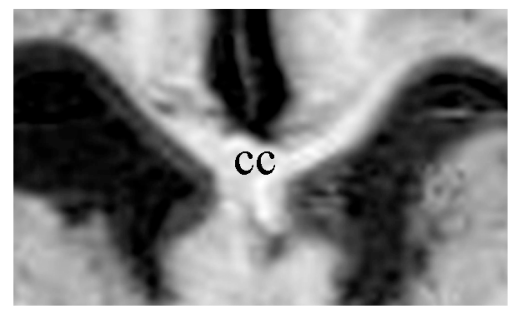

GW 15

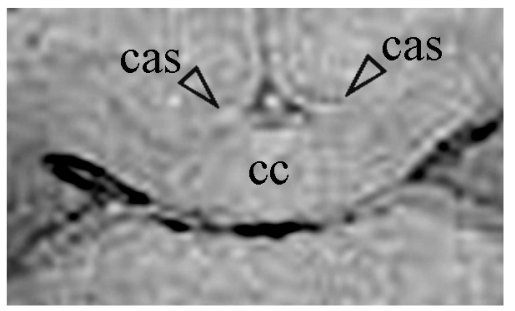


A. Development of calcarine sulcus
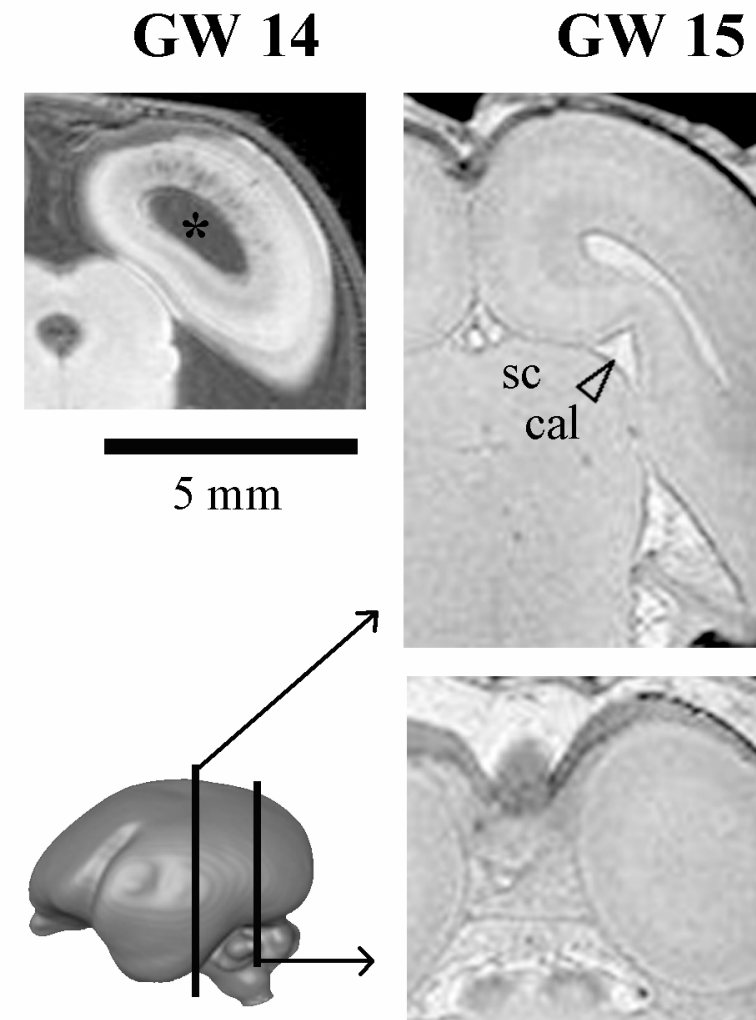

B. Development of occipitotemporal sulcus
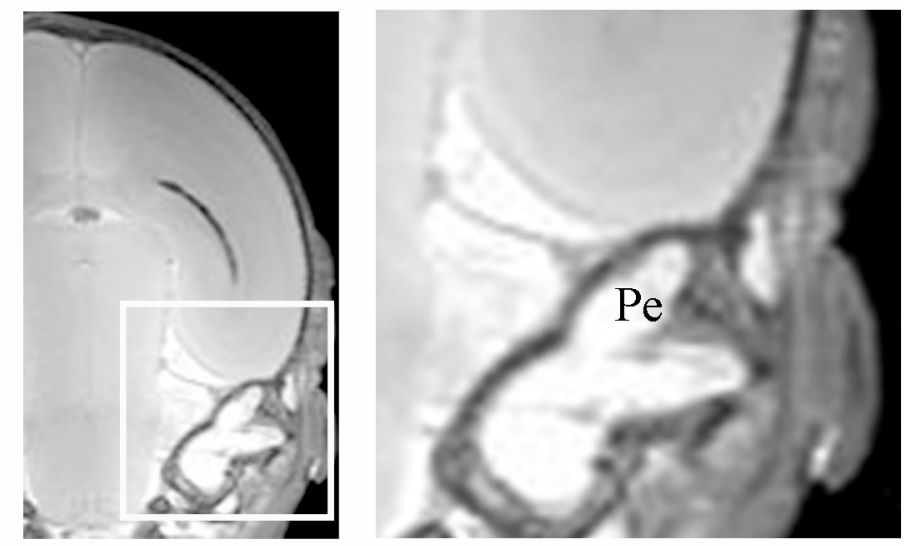

GW 18
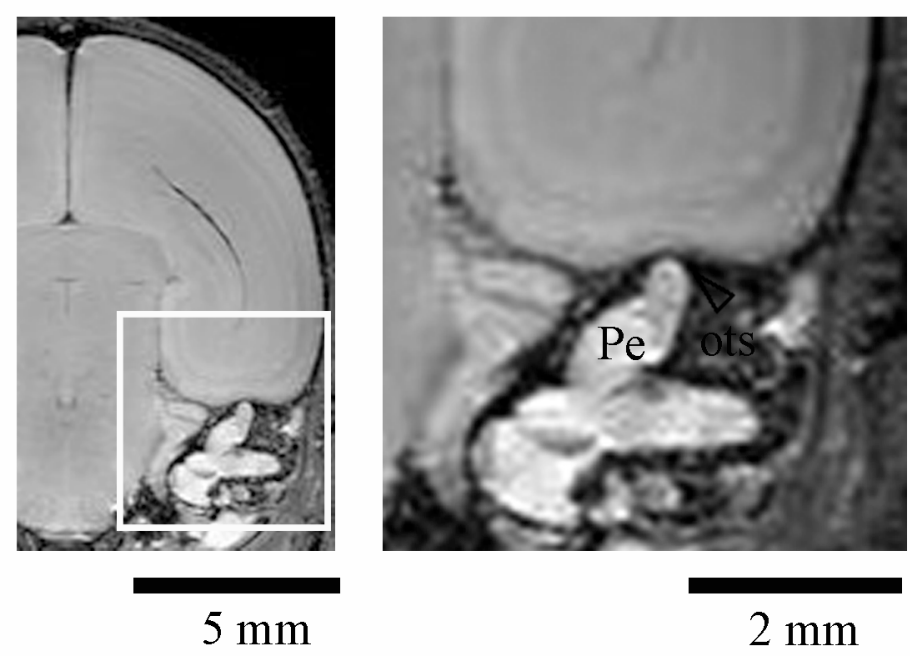
A.

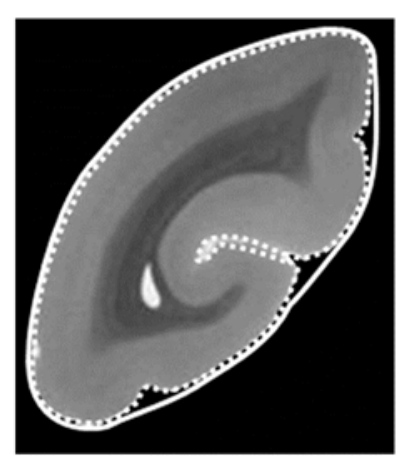

$\mathrm{GI}=\frac{\text { inner contour }(-\cdots \cdot \cdot)}{\text { outer contour }(-)}$
B.

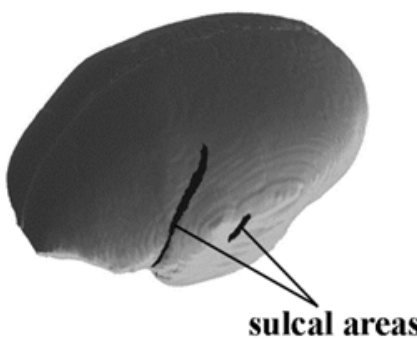

closed surface area $(\square)$

()

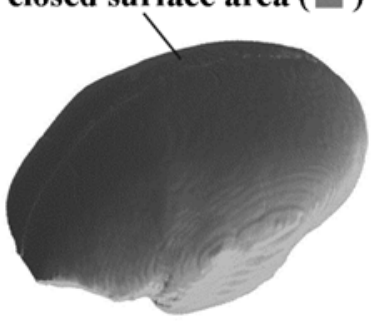

$\mathrm{SI}=\frac{\text { sulcal areas }(\square)}{\text { closed surface area }(\square)}$

C.

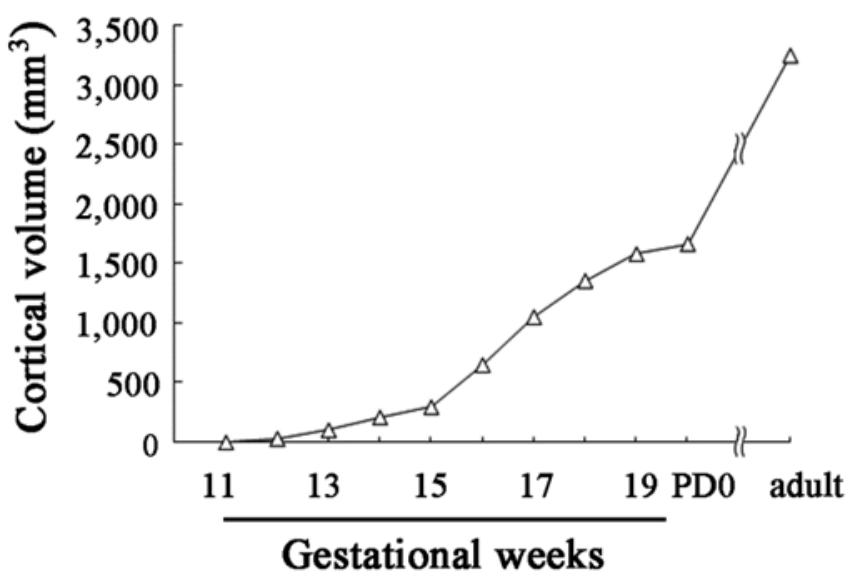

E.

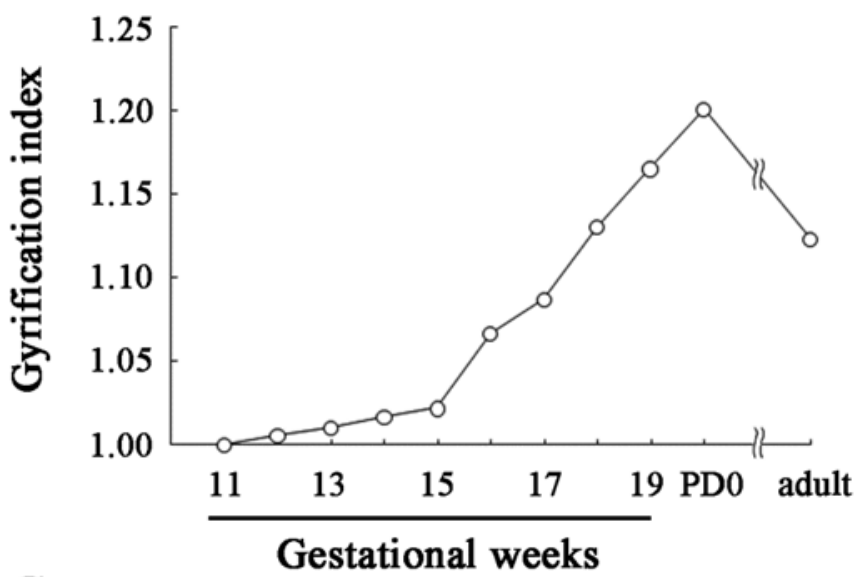

G.

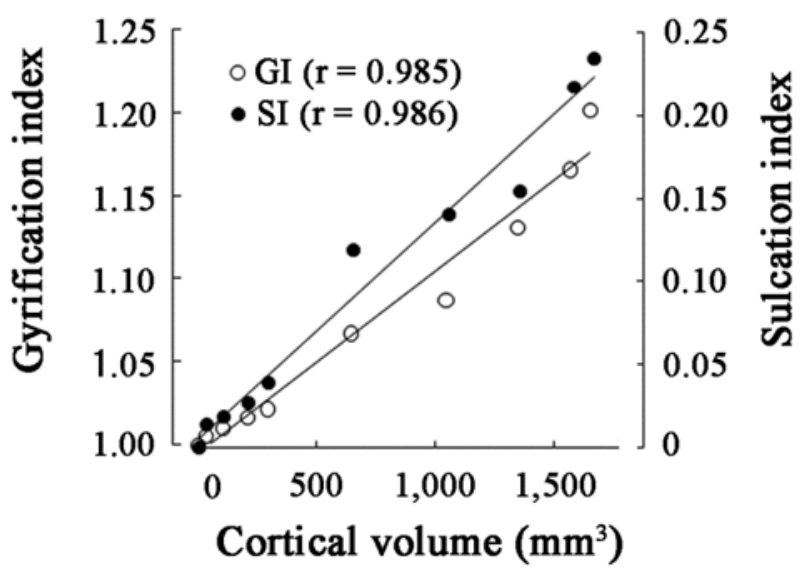

D.

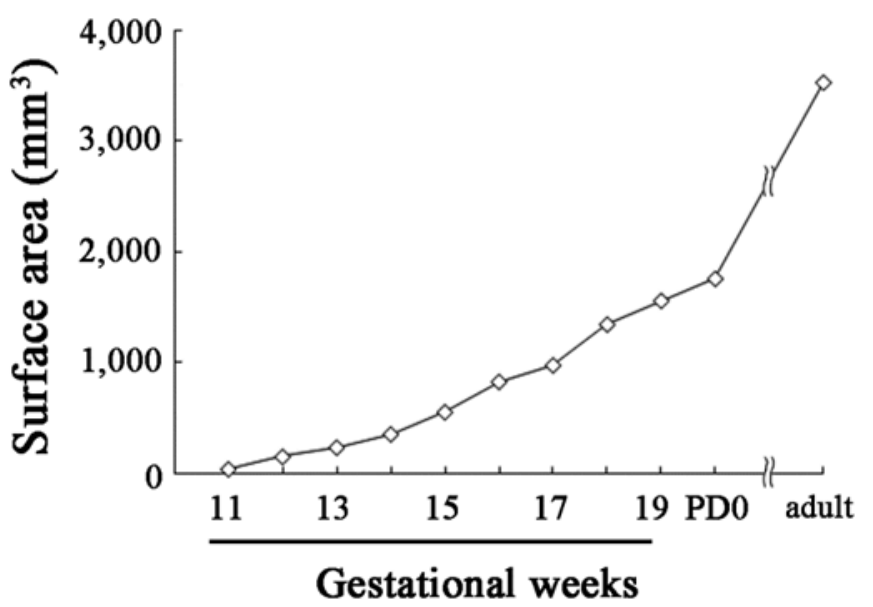

F.

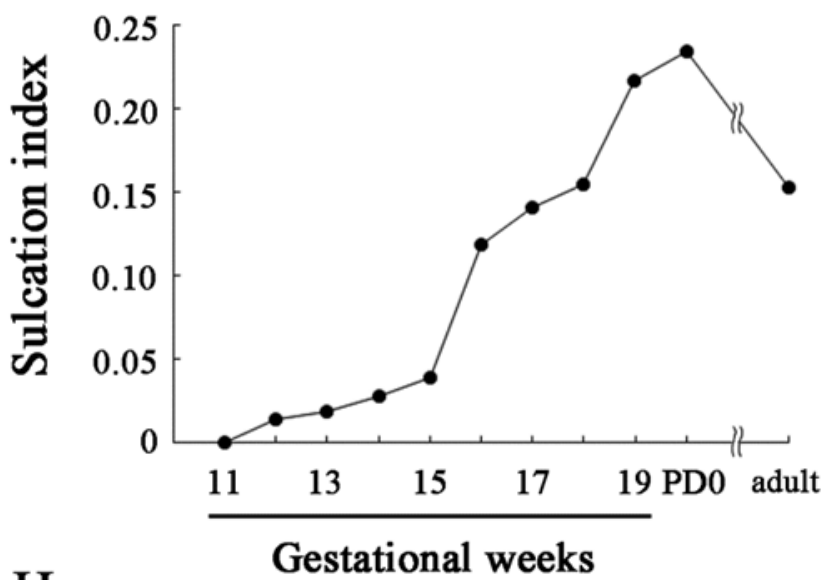

H.

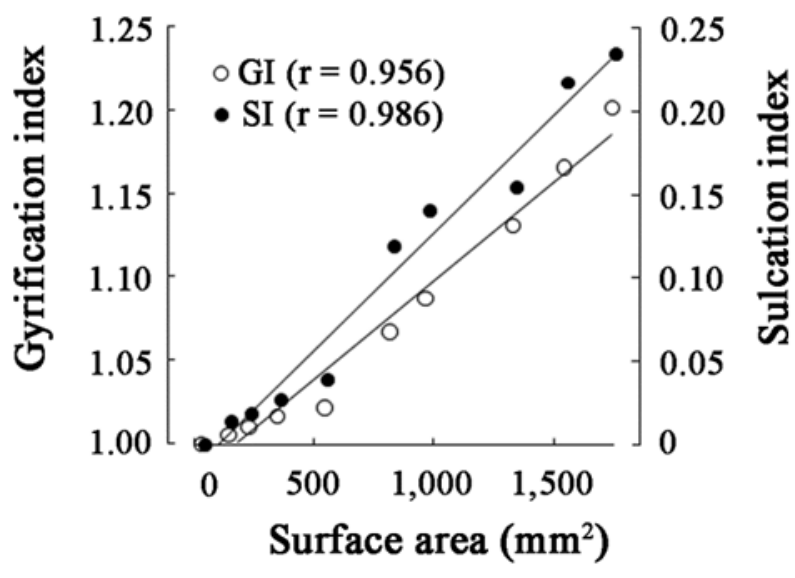



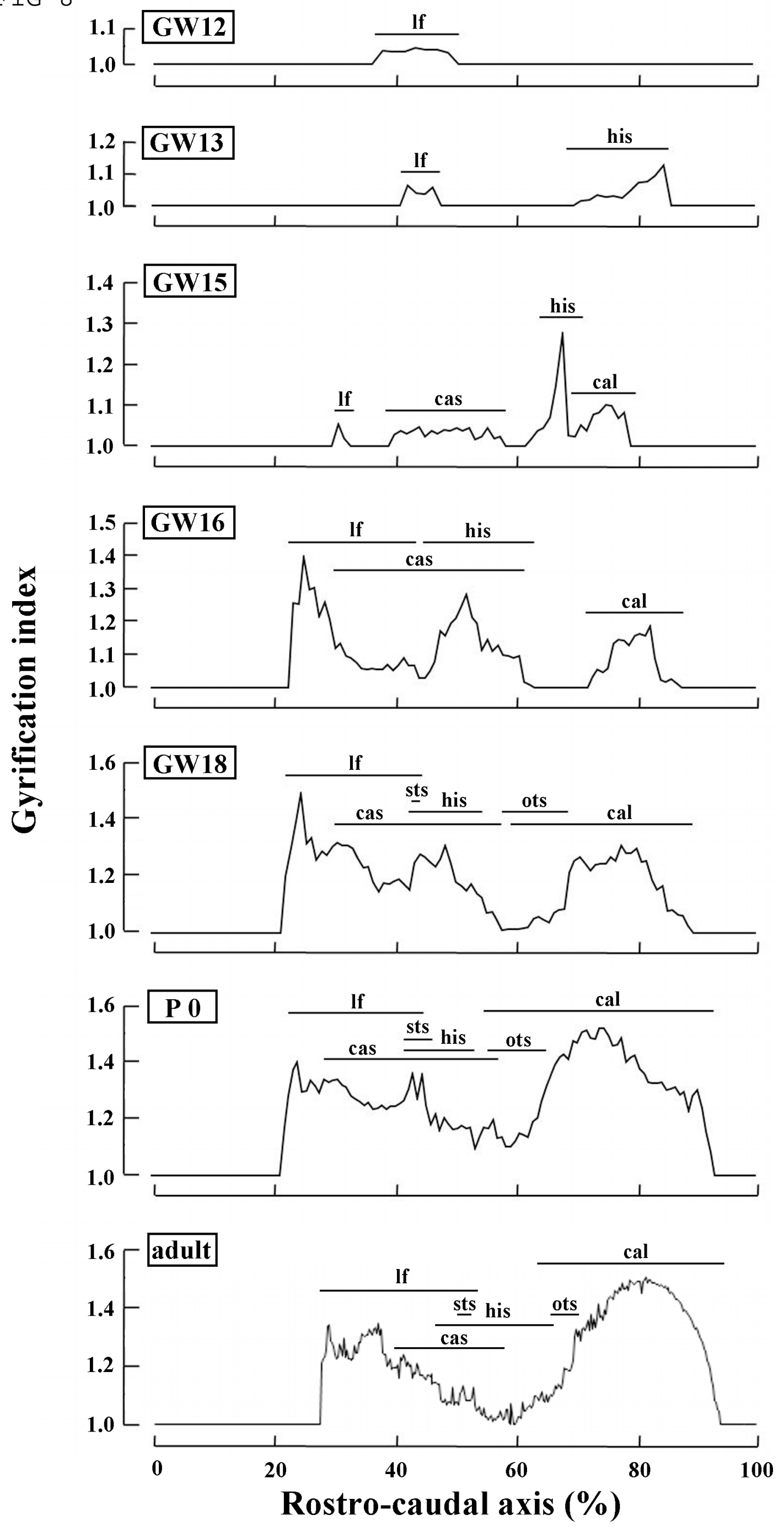
A. Presumptive calcarine sulcal regions

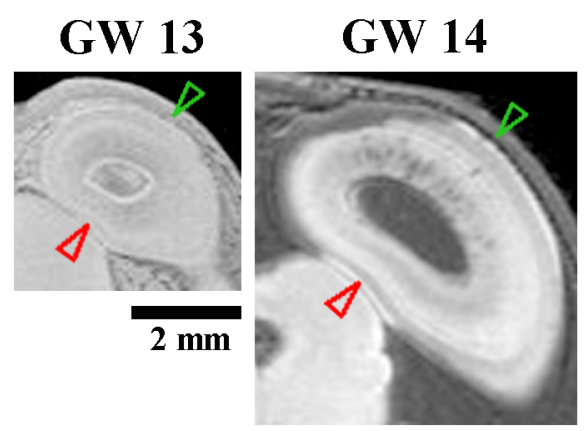

$\Delta$ presumptive calcarine sulcal region $\Delta$ calcarine sulcus

$\Delta$ non-sulcal region

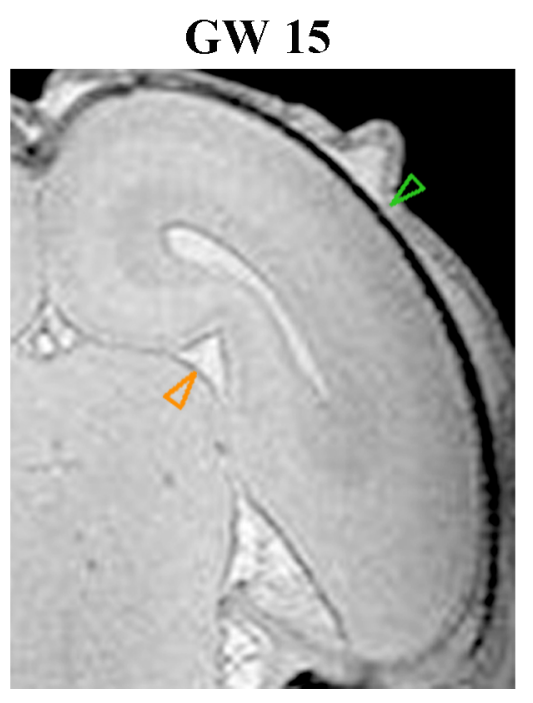

B. Thickness of cerebral wall

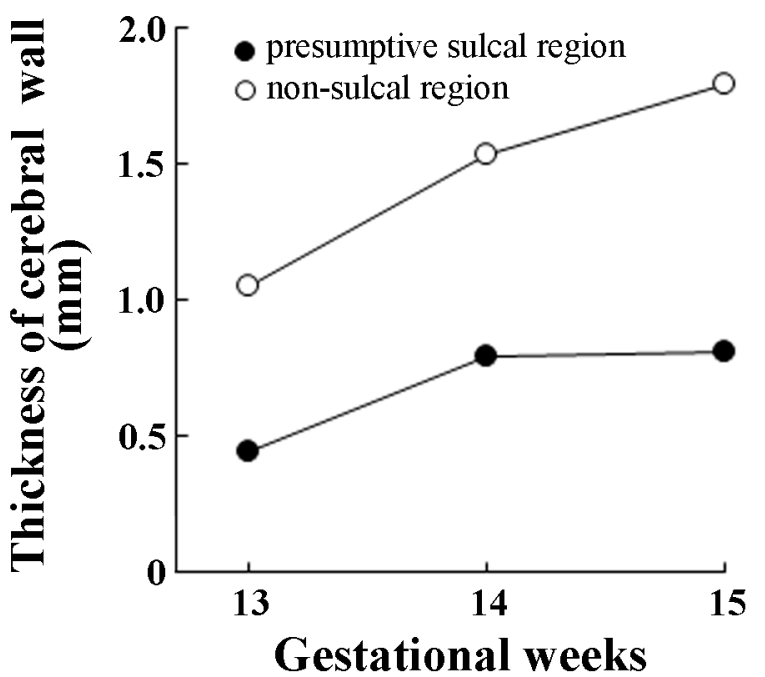

C. 3D maps of cortical thickness
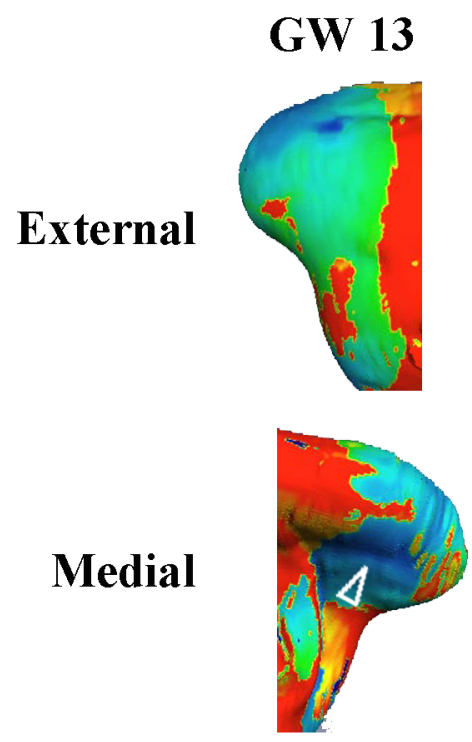

GW 14
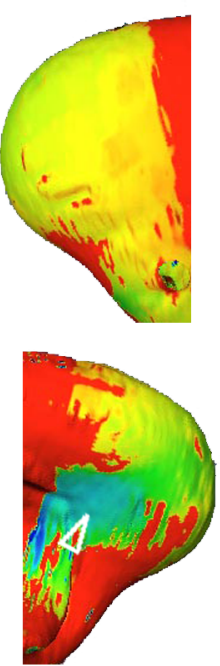

GW 15
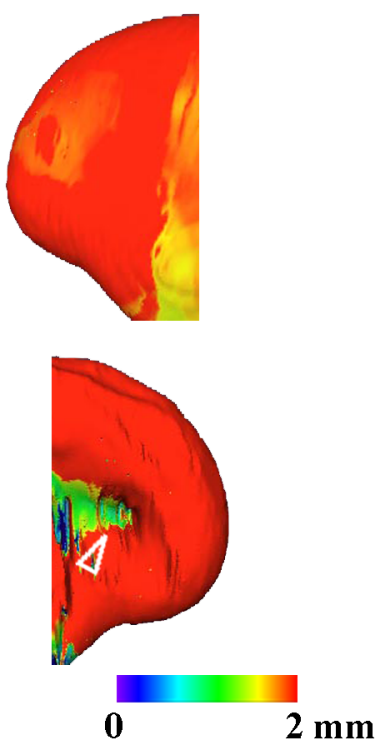

GW 13 (low-range)

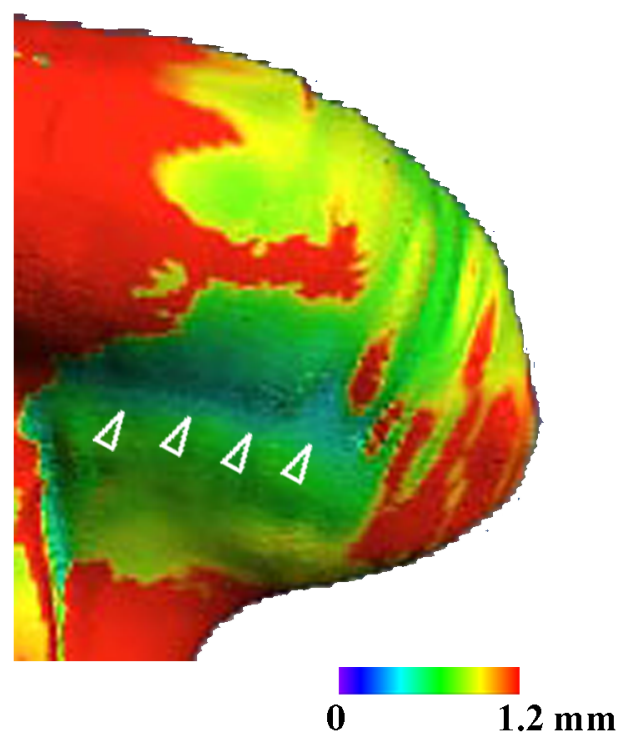

\section{Premature layers of cerebral wall (GW 13)}

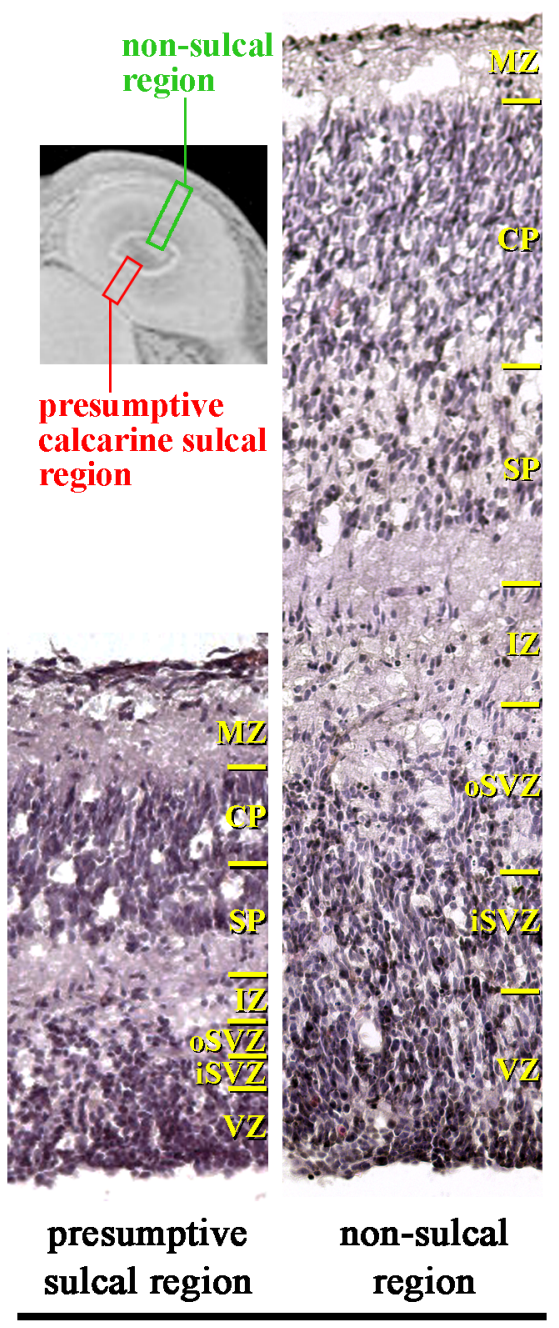

HE staining
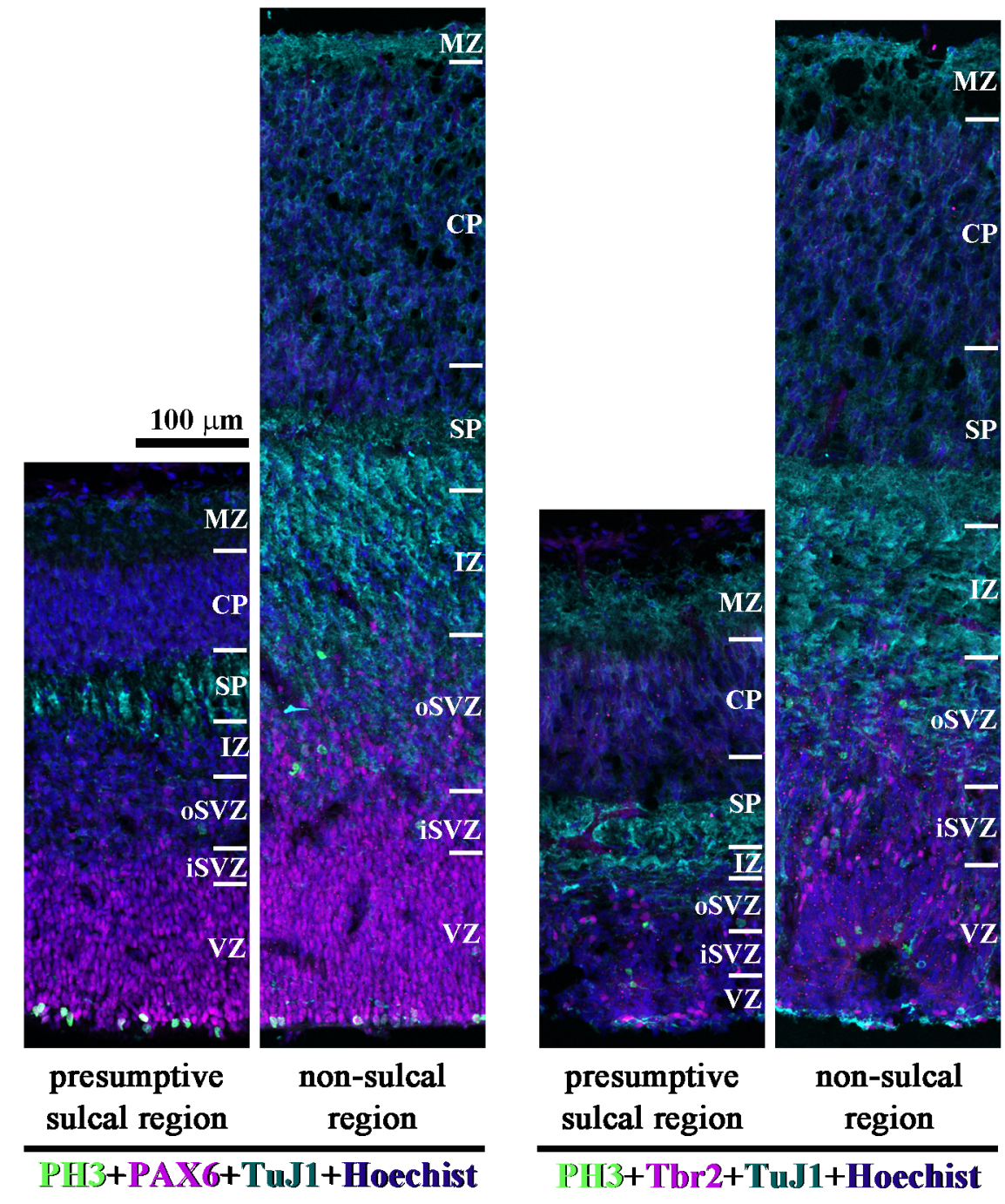

P[I3+Tbr2+TuJ1+Hoechist 
Table 1. Specimen and imaging specifications.

\begin{tabular}{|c|c|c|c|}
\hline Gestational weeks/postnatal agess & CRL $[\mathrm{mm}]$ & RF coil & Isotropic resolution [lm] \\
\hline GW8 & 6.2 & CryoProbe & 30 \\
\hline GW9 & 7.8 & CryoProbe & 30 \\
\hline GW10 & 10.8 & CryoProbe & 30 \\
\hline GW11 & 15.8 & 22 mm I.D. volume coil & 40 \\
\hline GW12 & 26.6 & 22 mm I.D. volume coil & 55 \\
\hline GW13 & 36.7 & 22 mm I.D. volume coil & 55 \\
\hline GW14 & 44.5 & 38 mm I.D. volume coil & 55 \\
\hline GW15 & 52.8 & 38 mm I.D. volume coil & 75 \\
\hline GW16 & 61.8 & 38 mm I.D. volume coil & 75 \\
\hline GW17 & 64.5 & 38 mm I.D. volume coil & 100 \\
\hline GW18 & 68.7 & 38 mm I.D. volume coil & 100 \\
\hline GW19 & 86.3 & 38 mm I.D. volume coil & 100 \\
\hline PD0 & - & 38 mm I.D. volume coil & 100 \\
\hline Adult & & & \\
\hline
\end{tabular}

This table was reproduced from our previous study (Hikishima et al., 2013). 
Table 2. Criteria for identification of premature layer of cortical wall of marmoset fetuses by Kelava et al. (2012)

\begin{tabular}{ll}
\hline Premature layers & Pattern of nuclear density and orientation \\
\hline Marginal zone (MZ) & most superficial layer with a sparse cell density \\
Cortical plate (CP) & high density of radial-oriented nuclei \\
Subplate (SP) & segregated from adjacent layers by nuclear pattern \\
Intermediate zone (IZ) & identified as cell-sparse layer \\
Outer subventricular zone (oSVZ) & high density of more radial-oriented nuclei (than iSVZ) \\
Inner subventricular zone (iSVZ) & lower density of nuclei with a less radial orientation \\
Ventricular zone (VZ) & high density of radial-oriented nuclei \\
\hline
\end{tabular}


Table 3. Variability of incidences of secondary sulci in adult marmosets

\begin{tabular}{|c|c|c|c|c|c|c|}
\hline \multirow{2}{*}{ Sexes } & \multicolumn{2}{|c|}{ Central sulcus } & \multicolumn{2}{|c|}{ Parietooccipital sulcus } & \multicolumn{2}{|c|}{ Cingulate sulcus } \\
\hline & Right & Left & Right & Left & Right & Left \\
\hline Males $(\mathrm{n}=10)$ & 7/10(70.0\%) & $4 / 10(40.0 \%)$ & $5 / 10(50.0 \%)$ & $5 / 10(50.0 \%)$ & $6 / 10(60.0 \%)$ & $7 / 10(70.0 \%)$ \\
\hline Females $(n=12)$ & $6 / 12(50.0 \%)$ & $6 / 12(50.0 \%)$ & $7 / 12(58.3 \%)$ & $6 / 12(50.0 \%)$ & $9 / 12(75.0 \%)$ & $9 / 12(75.0 \%)$ \\
\hline
\end{tabular}

No statistical differences were detected in either left/right side or sex by Z-test. 
Table 4. Thickness of oSVZ in presumptive sulcal and non-sulcal regions of occipital cortex in marmoset fetuses on GW 13

\begin{tabular}{lccc|}
\hline Regions & $\begin{array}{c}\text { Thickness of } \\
\text { oSVZ }(\mu \mathrm{m})\end{array}$ & $\begin{array}{c}\text { Thickness of } \\
\text { cortical wall }(\mu \mathrm{m})\end{array}$ & $\begin{array}{c}\text { \% of oSVZ } \\
\text { to cerebral wall }\end{array}$ \\
\hline $\begin{array}{l}\text { Presumptive calcarine sulcal region } \\
\text { (Upper part of medial surface) }\end{array}$ & 57.5 & 384.0 & 14.8 \\
$\begin{array}{l}\text { Non sulcal region } \\
\text { (External surface) }\end{array}$ & 206.4 & 865.2 & 23.9 \\
\hline
\end{tabular}

$\mathrm{n}=2$ 
Table 5. Comparison of chronologies of primary sulcal emergence among three primate species.

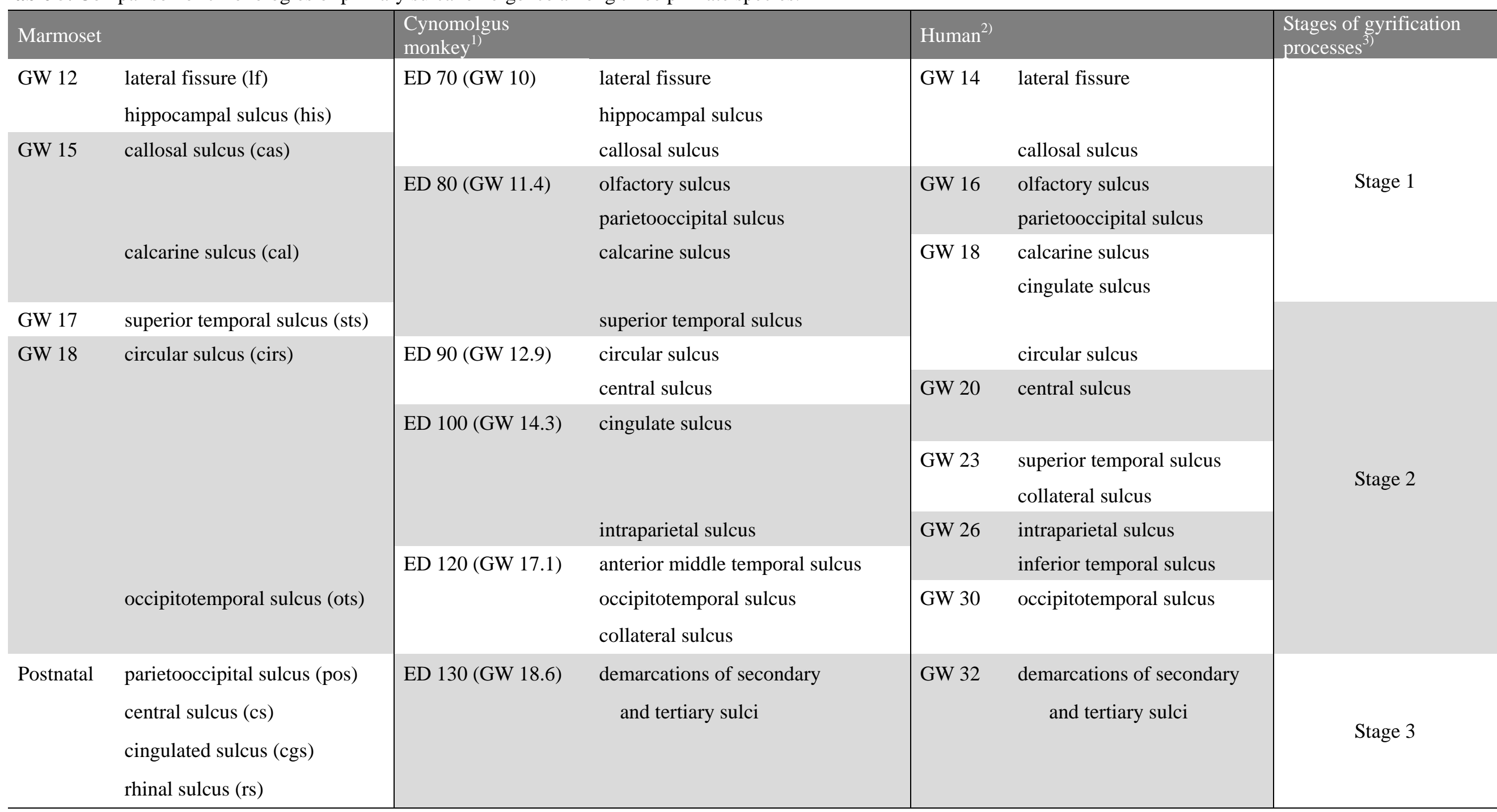

\footnotetext{
1) according to Sawada et al. (2009)

2) according to Chi et al. (1977)

3) proposed by our previous paper (Sawada et al. 2012a)
}

Cerebral sulci on same lines are anatomically identical among common marmosets, cynomolgus monkeys and humans. 